\title{
Stellar parameters of early-M dwarfs from ratios of spectral features at optical wavelengths ${ }^{\star, \star \star, \star \star \star}$
}

\author{
J. Maldonado ${ }^{1}$, L. Affer ${ }^{1}$, G. Micela ${ }^{1}$, G. Scandariato ${ }^{2}$, M. Damasso ${ }^{3}$, B. Stelzer ${ }^{1}$, M. Barbieri ${ }^{4}$, L. R. Bedin ${ }^{4}$, \\ K. Biazzo ${ }^{2}$, A. Bignamini ${ }^{5}$, F. Borsa ${ }^{6}$, R. U. Claudi ${ }^{4}$, E. Covino ${ }^{7}$, S. Desidera ${ }^{4}$, M. Esposito ${ }^{8,9}$, R. Gratton ${ }^{4}$, \\ J. I. González Hernández ${ }^{8,9}$, A. F. Lanza ${ }^{2}$, A. Maggio ${ }^{1}$, E. Molinari1 ${ }^{10,11}$, I. Pagano ${ }^{2}$, M. Perger ${ }^{12}$, I. Pillitteri ${ }^{1}$, \\ G. Piotto ${ }^{13,4}$, E. Poretti ${ }^{6}$, L. Prisinzano ${ }^{1}$, R. Rebolo ${ }^{8,9}$, I. Ribas ${ }^{12}$, E. Shkolnik ${ }^{14}$, J. Southworth ${ }^{15}$, \\ A. Sozzetti ${ }^{3}$, and A. Suárez Mascareño 8,9 \\ 1 INAF-Osservatorio Astronomico di Palermo, Piazza Parlamento 1, 90134 Palermo, Italy \\ e-mail: jmaldonado@astropa.inaf.it \\ 2 INAF-Osservatorio Astrofisico di Catania, via S. Sofia 78, 95123 Catania, Italy \\ 3 INAF-Osservatorio Astrofisico di Torino, via Osservatorio 20, 10025 Pino Torinese, Italy \\ 4 INAF-Osservatorio Astronomico di Padova, Vicolo Osservatorio 5, 35122 Padova, Italy \\ 5 INAF-Osservatorio Astronomico di Trieste, via Tiepolo 11, 34143 Trieste, Italy \\ 6 INAF-Osservatorio Astronomico di Brera, via E. Bianchi 46, 23807 Merate (LC), Italy \\ 7 INAF-Osservatorio Astronomico di Capodimonte, via Moiariello, 16, 80131 Naples, Italy \\ ${ }^{8}$ Instituto de Astrofísica de Canarias, 38205 La Laguna, Tenerife, Spain \\ 9 Universidad de La Laguna, Dpto. Astrofísica, 38206 La Laguna, Tenerife, Spain \\ 10 Fundación Galileo Galilei - INAF, Rambla José Ana Fernandez Pérez 7, 38712 Breña Baja, TF, Spain \\ 11 INAF-IASF Milano, via Bassini 15, 20133 Milano, Italy \\ 12 Institut de Ciéncies de 1'Espai (CSIC-IEEC), Campus UAB, Facultat de Ciéncies, Torre C5 parell, 2a planta, 08193 Bellaterra, \\ Spain \\ 13 Dip. di Fisica e Astronomia Galileo Galilei - Università di Padova, Vicolo dell'Osservatorio 2, 35122 Padova, Italy \\ 14 Lowell Observatory, 1400 W. Mars Hill Road, Flagstaff, AZ 86001, USA \\ 15 Astrophysics Group, Keele University, Staffordshire, ST5 5BG, UK
}

Received 3 February 2015 / Accepted 10 March 2015

\section{ABSTRACT}

\begin{abstract}
Context. Low-mass stars have been recognised as promising targets in the search for rocky, small planets with the potential of supporting life. As a consequence, Doppler search programmes using high-resolution spectrographs like HARPS or HARPS-N are providing huge quantities of optical spectra of $\mathrm{M}$ dwarfs. However, determining the stellar parameters of $\mathrm{M}$ dwarfs using optical spectra has proven to be challenging.

Aims. We aim to calibrate empirical relationships to determine accurate stellar parameters for early-M dwarfs (spectral types M0M4.5) using the same spectra as those that are used for radial velocity determinations, without the necessity of acquiring IR spectra or relying on atmospheric models and/or photometric calibrations.

Methods. Our methodology consists of using ratios of pseudo-equivalent widths of spectral features as a temperature diagnostic, a technique frequently used in solar-type stars. Stars with effective temperatures obtained from interferometric estimates of their radii are used as calibrators. Empirical calibrations for the spectral type are also provided. Combinations of features and ratios of features are used to derive calibrations for the stellar metallicity. Our methods are then applied to a large sample of M dwarfs that are currently being observed in the framework of the HARPS GTO search for extrasolar planets. The derived temperatures and metallicities are used together with photometric estimates of mass, radius, and surface gravity to calibrate empirical relationships for these parameters. Results. A long list of spectral features in the optical spectra of early-M dwarfs was identified. This list shows that the pseudoequivalent width of roughly $43 \%$ of the features is strongly anticorrelated with the effective temperature. The correlation with the stellar metallicity is weaker. A total of 112 temperature sensitive ratios were identified and calibrated over the range 3100-3950 K, providing effective temperatures with typical uncertainties of about $70 \mathrm{~K}$. Eighty-two ratios of pseudo-equivalent widths of features were calibrated to derive spectral types within 0.5 subtypes for stars with spectral types between $\mathrm{K} 7 \mathrm{~V}$ and M4.5V. We calibrated 696 combinations of the pseudo-equivalent widths of individual features and temperature-sensitive ratios for the stellar metallicity over a metallicity range from -0.54 to $+0.24 \mathrm{dex}$, with estimated uncertainties in the range of $0.07-0.10$ dex. We provide our own empirical calibrations for stellar mass, radius, and surface gravity. These parameters depend on the stellar metallicity. For a given effective temperature, lower metallicities predict lower masses and radii as well as higher gravities.
\end{abstract}

Key words. techniques: spectroscopic - stars: late-type - stars: low-mass - stars: fundamental parameters

\footnotetext{
$\star$ Based on data products from observations made with ESO Telescopes at the La Silla Paranal Observatory under programmes ID 072.C0488(E), 082.C-0718(B), 085.C-0019(A), 180.C-0886(A), 183.C-0437(A), and 191.C-0505(A), as well as data from the Italian Telescopio Nazionale Galileo (TNG) Archive (programmes ID CAT-147, and A27CAT_83).

$\star \star$ Our computational codes including the full version of Tables 2,4 , and 6 are only available at the CDS via anonymous ftp to cdsarc.u-strasbg.fr (130.79.128.5) or via http://cdsarc.u-strasbg.fr/viz-bin/qcat? J/A+A/577/A132

$\star \star \star$ Appendix $\mathrm{A}$ is available in electronic form at http://www. aanda.org
} 


\section{Introduction}

Ratios of equivalent widths or central depths of absorption lines with different excitation potentials have been widely used as temperature indicators in different types of stars, including solartype (e.g. Gray \& Johanson 1991; Gray 1994; Caccin et al. 2002; Kovtyukh et al. 2003; Biazzo et al. 2007; Montes et al. 2007; Sousa et al. 2010; Datson et al. 2012, 2014, 2015), giant stars (e.g. Gray 1989; Strassmeier \& Schordan 2000), and supergiants (e.g. Kovtyukh \& Gorlova 2000). To the best of our knowledge, this technique has not been extended to the lowmass star regime, however, most likely because of the difficulties in analysing their optical spectrum, which is mainly covered by molecular bands (in particular $\mathrm{TiO}$ and water) that blend or hide most of the atomic lines commonly used in the spectral analysis of solar-type stars. Furthermore, $M$ dwarfs are intrinsically faint in the optical.

The accurate determination of stellar parameters for $\mathrm{M}$ dwarfs has proven to be a difficult task. Some photometric calibrations of the stellar metallicity are based on optical and near-infrared photometry, a technique pioneered by Bonfils et al. (2005) and updated by Johnson \& Apps (2009), Schlaufman \& Laughlin (2010) and more recently by Neves et al. (2012, hereafter NE12), although they require accurate parallaxes and magnitudes, which are usually only available for nearby and bright stars. Another common technique for characterising M dwarf metallicities is based on the use of spectroscopic indices that measure the relative strength of the $\mathrm{TiO}$ molecular band with respect to the $\mathrm{CaH}$ molecular bands near $7000 \AA$ (Lépine et al. 2007, 2013; Dhital et al. 2012). Since the spectral energy distribution of $\mathrm{M}$ dwarfs peaks at infrared wavelengths, some previous works have performed a search for spectral features and indices in this spectral region. In particular, Rojas-Ayala et al. (2012) used the equivalent width of the $\mathrm{Na}$ I and $\mathrm{Ca}$ I triplet and the $\mathrm{H}_{2} \mathrm{O}-\mathrm{K} 2$ index in the $K$ band of the spectra. This methodology has also been applied by Terrien et al. (2012) and Mann et al. (2013a), providing calibrations for $\mathrm{H}$ and $\mathrm{J} /$ optical spectral bands, respectively. Large samples of $M$ dwarfs have been characterised by means of near-infrared indices in the recent works by Newton et al. (2014) and Gaidos et al. (2014). On the other hand, spectral synthesis has been tested in several works, usually on small numbers of stars and mainly focusing on strong atomic lines or on spectral windows known to be less affected by molecular lines (Woolf \& Wallerstein 2005; Bean et al. 2006; Maness et al. 2007; Önehag et al. 2012; Rajpurohit et al. 2014).

Concerning the effective temperature, very few $\mathrm{M}$ dwarfs are bright enough for a direct measurement of their radii (e.g. Boyajian et al. 2012, hereafter BO12), a technique pioneered by Ségransan et al. (2003). The most common technique for determining the effective temperature of an $\mathrm{M}$ dwarf is to compare observed spectra with model atmospheres (e.g. Lépine et al. 2013; Gaidos et al. 2014). Casagrande et al. (2008, hereafter CA08) provided optical/near-infrared photometric calibrations based on an extension of the infrared flux method (IRFM) for FGK dwarfs from Casagrande et al. (2006) to M dwarfs. However, significant systematic differences between temperatures based on CA08 calibrations and temperatures based on interferometric radii measurements have recently been noted by Mann et al. (2015).

Despite the intrinsic difficulties in their characterisation, low-mass stars are today at the centre of the search for small, rocky planets with the potential capability of hosting life (e.g. Dressing \& Charbonneau 2013; Sozzetti et al. 2013). In particular, the radial velocity searches currently ongoing with HARPS at La Silla and in the framework of the Global Architecture of Planetary Systems project ${ }^{1}$ (GAPS; Covino et al. 2013) at the Telescopio Nazionale Galileo (TNG) with HARPS-N produce a large quantity of spectra with high resolution and a high signalto-noise ratio. Exoplanet searches would certainly benefit from a methodology for determining accurate stellar parameters using the same spectra that are being used for the radial velocity determinations, that is, without the necessity of observing at infrared facilities (usually from space) or relying on atmospheric models. Following this line of reasoning, a methodology for characterising $\mathrm{M}$ dwarfs from high-resolution optical spectra by using pseudo-equivalent widths of features has been presented in a recent work by Neves et al. (2014, hereafter NE14).

The idea of the pseudo-equivalent width can be further exploited to calibrate empirical relationships for M dwarfs. This is the goal of this paper, in which we present a large database of empirical calibrations of spectral features, ratios of features, and combinations of both with the aim of deriving $T_{\text {eff }}$, spectral type, and metallicity, for early-M dwarf stars by using optical HARPS and HARPS-N spectra (wavelength range 383-693 nm). Unlike NE14, we take as reference temperature scale the one provided by BO12 and not that of CA08. We also apply our method to derive spectral types. Furthermore, we provide relations for stellar masses, radii, and surface gravities so that these quantities can be obtained without using parallaxes or photometry. We use our methods to characterise in an homogeneous and coherent way a sample of the $\mathrm{M}$ dwarfs that are currently being monitored in the HARPS GTO radial velocity programme (Bonfils et al. 2013). Late-M stars are excluded from this study since an exoplanet search around these stars is difficult at optical wavelengths.

This paper is organised as follows: Sect. 2 describes the spectroscopic data used in this work. Section 3 describes our methodology and how empirical calibrations for the main stellar parameters are built. These calibrations are then applied to a large sample of stars, and results are compared with other techniques in Sect. 4. The derived temperatures and metallicities are used to build empirical calibrations for stellar masses, radii, and gravities in Sect. 5. Our conclusions follow in Sect. 6.

\section{Spectroscopic data}

This work makes use of HARPS (Mayor et al. 2003) and HARPS-N (Cosentino et al. 2012) spectra mostly taken from archive. Specifically, the data are taken from i) the ESOpipeline-processed FEROS and HARPS archive $^{2}$; ii) the ESO Science Data products Archive ${ }^{3}$; and iii) the TNG Archive ${ }^{4}$. The corresponding ESO and TNG programme IDs are listed in the footnote to the paper title. In addition to the data from archive, some HARPS-N spectra have been provided by the GAPS team.

The instrumental setup of HARPS and HARPS-N is almost identical, which means that data from the two spectrographs can be used together. The spectra cover the range 383-693 nm (HARPS-N) and 378-691 nm (HARPS). Both instruments provide a resolving power of $R \sim 115000$. The spectra are provided already reduced using ESO/HARPS-N standard calibration pipelines. Typical values of the signal-to-noise ratio are between 50 and 90 (measured at $\sim 5500 \AA$ A). Wavelengths are on

\footnotetext{
1 http://www.oact.inaf.it/exoit/EXO-IT/Projects/ Entries/2011/12/27_GAPS.html

2 http://archive.eso.org/wdb/wdb/eso/repro/form

3 http://archive.eso.org/wdb/wdb/adp/phase3_spectral/

form?phase3_collection=HARPS

4 http://ia2.oats.inaf.it/archives/tng
} 
air. The spectra were corrected for radial velocity shifts by using the IRAF ${ }^{5}$ task dopcor. For this purpose, we used the accurate radial velocities (measured by applying the cross-correlation technique) that are provided with the reduced spectra.

\section{Methodology}

The optical spectrum of an M dwarf is a forest of lines and molecular bands that are usually heavily blended. Identifying individual lines (of moderate strength) or measuring equivalent widths in these forests is a difficult task. To overcome this limitation, we followed the idea outlined by NE14, and instead of considering equivalent widths or depths of lines, we considered pseudo-equivalent widths (EWs) of features. A feature can be a line or a blend of lines. The pseudo-equivalent width is defined as the traditional equivalent width, with the difference that it is not measured with respect to a continuum normalised to unity, but to the value of the flux between the peaks of the feature at each wavelength:

$E W=\sum \frac{F_{\mathrm{pp}}-F_{\lambda}}{F_{\mathrm{pp}}} \Delta \lambda$,

where $F_{\lambda}$ is the stellar flux, $F_{\mathrm{pp}}$ denotes the value of the flux between the peaks of the feature at each integration step, and $\Delta \lambda$ is the spectral wavelength step. An estimate of the uncertainty in the measured EWs is given by

$\sigma_{\mathrm{EW}}=\frac{\Delta \lambda}{\left\langle F_{\mathrm{pp}}\right\rangle} \sigma_{F_{\mathrm{pp}}}$

where $\left\langle F_{\mathrm{pp}}\right\rangle$ is the mean value of the flux between the peaks of the feature, and $\sigma_{F_{\mathrm{pp}}}$ its corresponding standard deviation. Figure 1 illustrates how the EWs are measured.

An initial list of 4224 features was built taking as reference the spectra of the star Gl 49, a M1.5V low-mass dwarf. Spectroscopic observations of this star were carried out within the framework of the GAPS project with HARPS-N. Only the red region of the spectra (5300-6900 $\AA$ ) was considered since the blue part of the optical spectrum of an M dwarf usually has a lower signal-to-noise ratio. Regions of the spectra affected by chromospheric activity and atmospheric absorption were avoided.

Figure 2 shows the effects of effective temperature and metallicity on the EWs measurement. In the left panel, a portion of the stellar spectra is shown for stars with similar metallicities, but different $T_{\text {eff }}$. The same portion of the spectra is shown in the right panel, this time for stars of similar $T_{\text {eff }}$, but different metallicities. A list of calibrators was built for each of the basic stellar parameters we considered ( $T_{\text {eff }}$, spectral type, and metallicity), following different criteria, as explained in the next subsections.

\subsection{Effective temperature}

The accuracy of the $T_{\text {eff }}$ derived from temperature-sensitive ratios is intimately tied to the accuracy of the temperature of the stars used as calibrators. We used as calibrators the sample of early-M dwarfs with angular sizes obtained with long-baseline interferometry to better than 5\% given in $\mathrm{BO} 12$.

BO12 list 22 low-mass stars with spectral-types equal to or cooler than $\mathrm{K} 5 \mathrm{~V}$, spanning a range of $T_{\text {eff }}$ between 3000

\footnotetext{
5 IRAF is distributed by the National Optical Astronomy Observatory, which is operated by the Association of Universities for Research in Astronomy, Inc., under contract with the National Science Foundation.
}

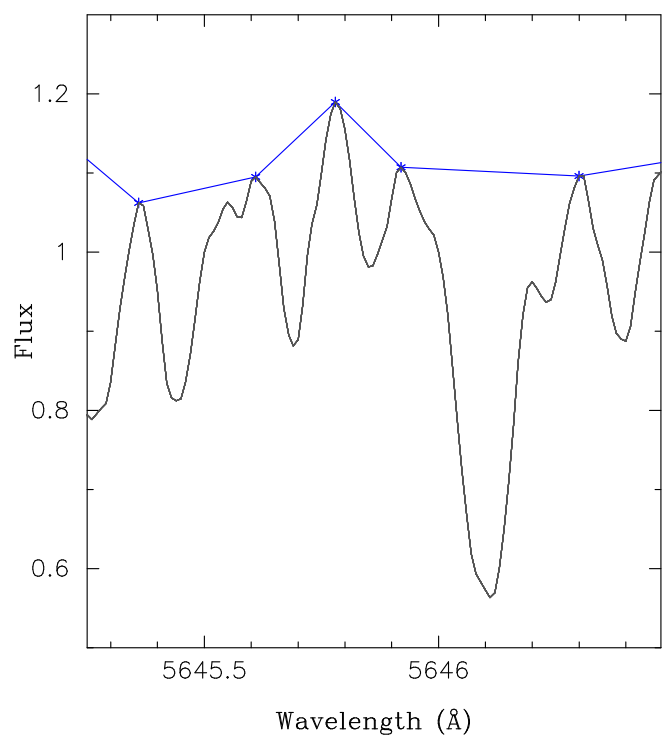

Fig. 1. Example of the EW measurement. The figure shows a portion of the spectrum of the star Gl 49. The flux between the peaks of the features is measured over the blue lines.

Table 1. Effective temperature calibration sample.

\begin{tabular}{lcc}
\hline \hline Star & SpT & $\begin{array}{c}T_{\text {eff }} \\
(\mathrm{K})\end{array}$ \\
\hline GJ338A & M0V & $3953 \pm 41$ \\
GJ205 & M1.5V & $3850 \pm 22$ \\
GJ880 & M1.5V & $3731 \pm 16$ \\
GJ176 & M2.5V & $3701 \pm 90$ \\
GJ887 & M0.5V & $3695 \pm 35$ \\
GJ526 & M1.5V & $3646 \pm 34$ \\
GJ15A & M1.5V & $3602 \pm 13$ \\
GJ412A & M1V & $3537 \pm 41$ \\
GJ436 & M3V & $3520 \pm 66$ \\
GJ581 & M2.5V & $3487 \pm 62$ \\
GJ725A & M3V & $3417 \pm 17$ \\
GJ699 & M4V & $3238 \pm 11$ \\
GJ876 & M5V & $3176 \pm 20$ \\
GJ725B & M3.5V & $3142 \pm 29$ \\
\hline
\end{tabular}

Notes. Effective temperatures are taken from Mann et al. (2013b) and spectral types from BO12. For GJ176 and GJ876, $T_{\text {eff }}$ values are adopted from Newton et al. (2015) and spectral types from the GJ catalogue (Gliese \& Jahreiß 1991).

and $4500 \mathrm{~K}$. HARPS spectra were obtained for seven stars from the ESO archive, whilst HARPS-N spectra were obtained for three stars from the TNG archive. HARPS-N spectra for two further stars have been provided by the GAPS team. To these stars, we added two more from the recent work by von Braun et al. (2014), who analyzed their stars in the same way as BO12 ${ }^{6}$.

Mann et al. (2013b) revised the temperature scale of BO12 because they noted a problem in the determination of the stellar bolometric flux. Although the temperature differences are relatively small, we used the set of updated temperatures. For the two stars taken from von Braun et al. (2014), updated $T_{\text {eff }}$ values computed in the same way as in Mann et al. (2013b) are provided in Newton et al. (2015). The final list of $T_{\text {eff }}$ calibrators includes 14 stars whose parameters are listed in Table 1.

\footnotetext{
6 The authors list three M dwarfs, but we were unable to obtain a HARPS or HARPS-N spectrum of Gl 649.
} 

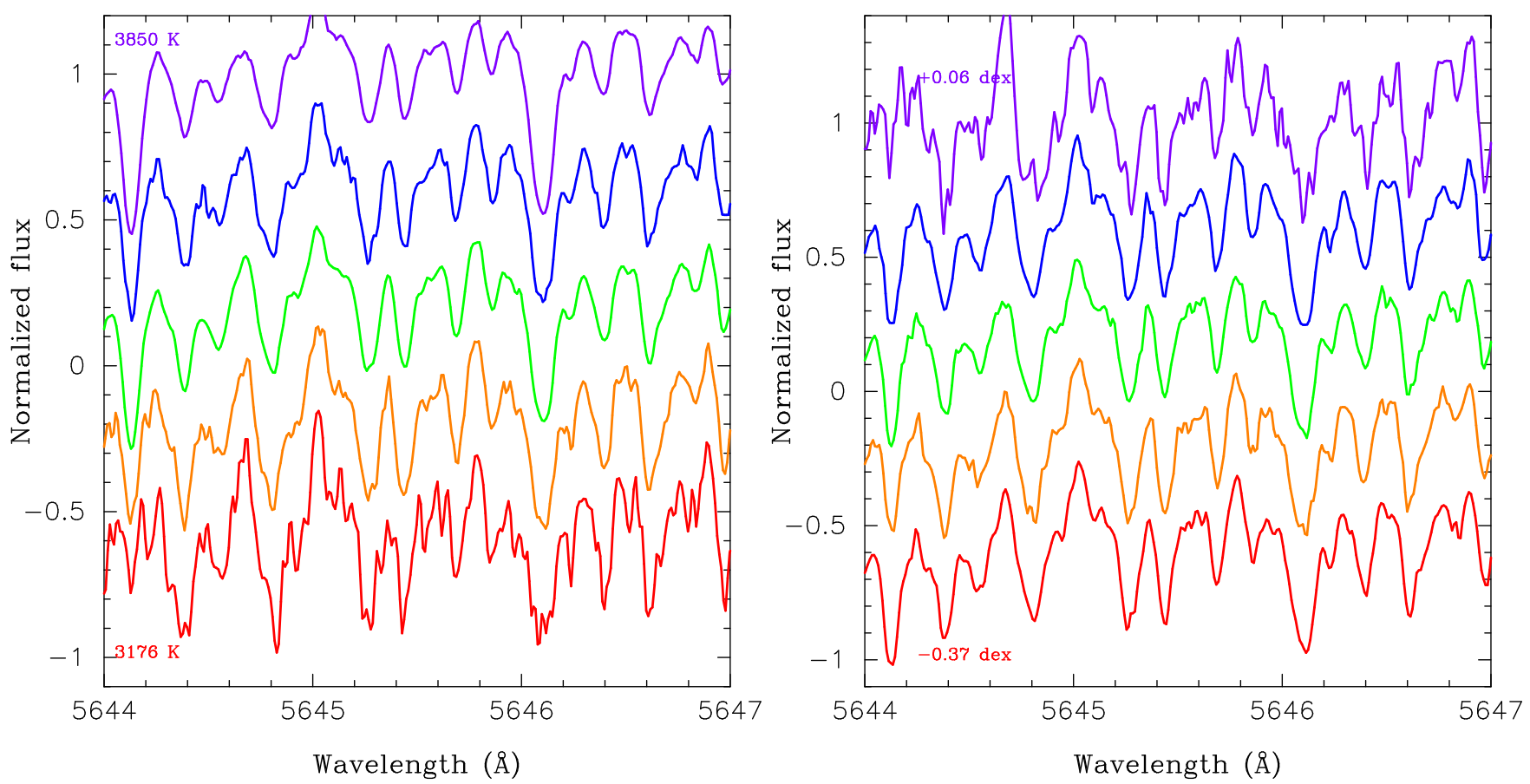

Fig. 2. Left: portion of the stellar spectrum of several $T_{\text {eff }}$ calibrators (see text for details) with similar metallicity (from -0.07 to +0.15 dex), but different effective temperature: $3850 \mathrm{~K}$ (purple), $3701 \mathrm{~K}$ (blue), $3646 \mathrm{~K}$ (green), $3520 \mathrm{~K}$ (orange), and $3176 \mathrm{~K}$ (red). Right: portion of the stellar spectrum of several metallicity calibrators (see text for details) with similar effective temperature (3450-3550 K), but different metallicity: $+0.06 \mathrm{dex}$ (purple), $+0.01 \mathrm{dex}$ (blue), $-0.11 \mathrm{dex}$ (green), $-0.20 \mathrm{dex}$ (orange), and $-0.37 \mathrm{dex}$ (red). For the sake of clarity, an offset of -0.40 in flux was applied between the spectra.

Starting from our initial list of 4224 identified features, the EW of all features were measured in all calibration stars. To avoid possible dependencies on microturbulence, rotation, or stellar metallicity, we rejected features with $E W<20 \mathrm{~m} \AA$ or $E W>120 \mathrm{~m} \AA$ in any of the calibration stars, thus excluding too weak and too strong features. We also rejected lines with relative errors $\left(\frac{\sigma_{\mathrm{EW}}}{E W}\right)$ larger than $2 \%$. For every possible ratio of features a Spearman correlation test was computed to check whether the ratio is temperature sensitive or not. The ratios were selected with the only condition that the features are separated by no more than $15 \AA$. This limit was set to avoid problems with scattered light correction or continuum normalisation. All ratios with a probability of correlation by chance lower than $2 \%$ were considered for further study ${ }^{7}$.

Following Kovtyukh et al. (2003), we fitted the $T_{\text {eff-ratio re- }}$ lationship to several functions for each considered EW ratio: a Hoerl function $\left(T_{\text {eff }}=a b^{r} \times r^{c}\right)$, a modified Hoerl function $\left(T_{\mathrm{eff}}=a b^{1 / r} \times r^{c}\right)$, a power-law function $\left(T_{\mathrm{eff}}=a \times r^{b}\right)$, an exponential law function $T_{\text {eff }}=a \times b^{r}$, and a logarithmic function $T_{\text {eff }}=a+b \times \ln (r)$, where $r=E W_{1} / E W_{2}$ is the ratio between the EW of two features. All fits were performed using a nonlinear least-squares fitting routine in IDL (MPFIT; Markwardt 2009) taking into account the uncertainties in $T_{\text {eff. }}$ For each calibration we selected the function that produced the smallest standard deviation, retaining only those calibrations with a standard deviation smaller than $75 \mathrm{~K}$. There are 112 selected temperaturesensitive ratios.

Given our relatively low number of calibrators, we additionally checked that the selected ratios are not correlated with $T_{\text {eff }}$

\footnotetext{
7 Ideally, for a ratio of lines to be temperature-sensitive, the excitation potential of the lines, $\chi$, must differ as much as possible. This is because the EWs of lines with higher $\chi$ change with $T_{\text {eff }}$ faster than those of lines with lower $\chi$ values (Gray 1994).
}

Table 2. Coefficients of our feature ratio-temperature relations.

\begin{tabular}{ccccccccc}
\hline \hline $\begin{array}{c}\lambda_{1} \\
(1)\end{array}$ & $\begin{array}{c}\Delta \lambda_{1} \\
(2)\end{array}$ & $\begin{array}{c}\lambda_{2} \\
(3)\end{array}$ & $\begin{array}{c}\Delta \lambda_{2} \\
(4)\end{array}$ & $\begin{array}{c}a \\
(5)\end{array}$ & $\begin{array}{c}b \\
(6)\end{array}$ & $\begin{array}{c}c \\
(7)\end{array}$ & $\begin{array}{c}\text { Func. }^{\dagger} \\
(8)\end{array}$ & $\begin{array}{c}\sigma(\mathrm{K}) \\
(9)\end{array}$ \\
\hline 6435.00 & 0.38 & 6432.01 & 0.34 & 4529.63 & 0.73 & -0.015 & $\mathrm{MH}$ & 49 \\
5720.46 & 0.33 & 5718.32 & 0.28 & 4842.67 & 0.66 & 0.89 & $\mathrm{H}$ & 53 \\
6226.67 & 0.35 & 6213.44 & 0.29 & 4350.09 & 0.86 & - & $\mathrm{E}$ & 54 \\
5708.61 & 0.30 & 5718.32 & 0.28 & 5122.92 & 0.634 & 0.92 & $\mathrm{H}$ & 54 \\
\hline
\end{tabular}

Notes. Columns (1) to (4) provide information about the features involved in the ratio (central wavelength and width in $\AA$ ), while Cols. (5) to (9) show the coefficients of the best-fitting relationships, their functional form, and the corresponding standard deviation of the $T_{\text {eff }}$ calibration. The same examples are shown in Fig. 3. The full table is available at the CDS. ${ }^{(\dagger)} \mathrm{H}$ : Hoerl; MH: modified Hoerl; PL: Power-law; E: Exponential; L: Logarithmic.

simply by coincidence. We created 1000 series of simulated random effective temperatures and errors, keeping the media and the standard deviation of the original data. For each series of simulated data we repeated our analysis and computed the number of suitable calibrations. The results show that in $98 \%$ of the simulations our methodology does not recover any suitable $T_{\text {eff }^{-}}$ calibration, whilst only in $0.8 \%$ of the simulations we obtain more than ten calibrations. We therefore conclude that it is very unlikely that our obtained $T_{\text {eff-ratios are correlated with } T_{\text {eff }} \text { just }}$ by chance.

Some examples of the selected temperature-sensitive ratios are shown in Fig. 3, whilst full details for the calibrations for the same examples can be found in Table 2. 
J. Maldonado et al.: Stellar parameters of early-M dwarfs from ratios of spectral features at optical wavelengths
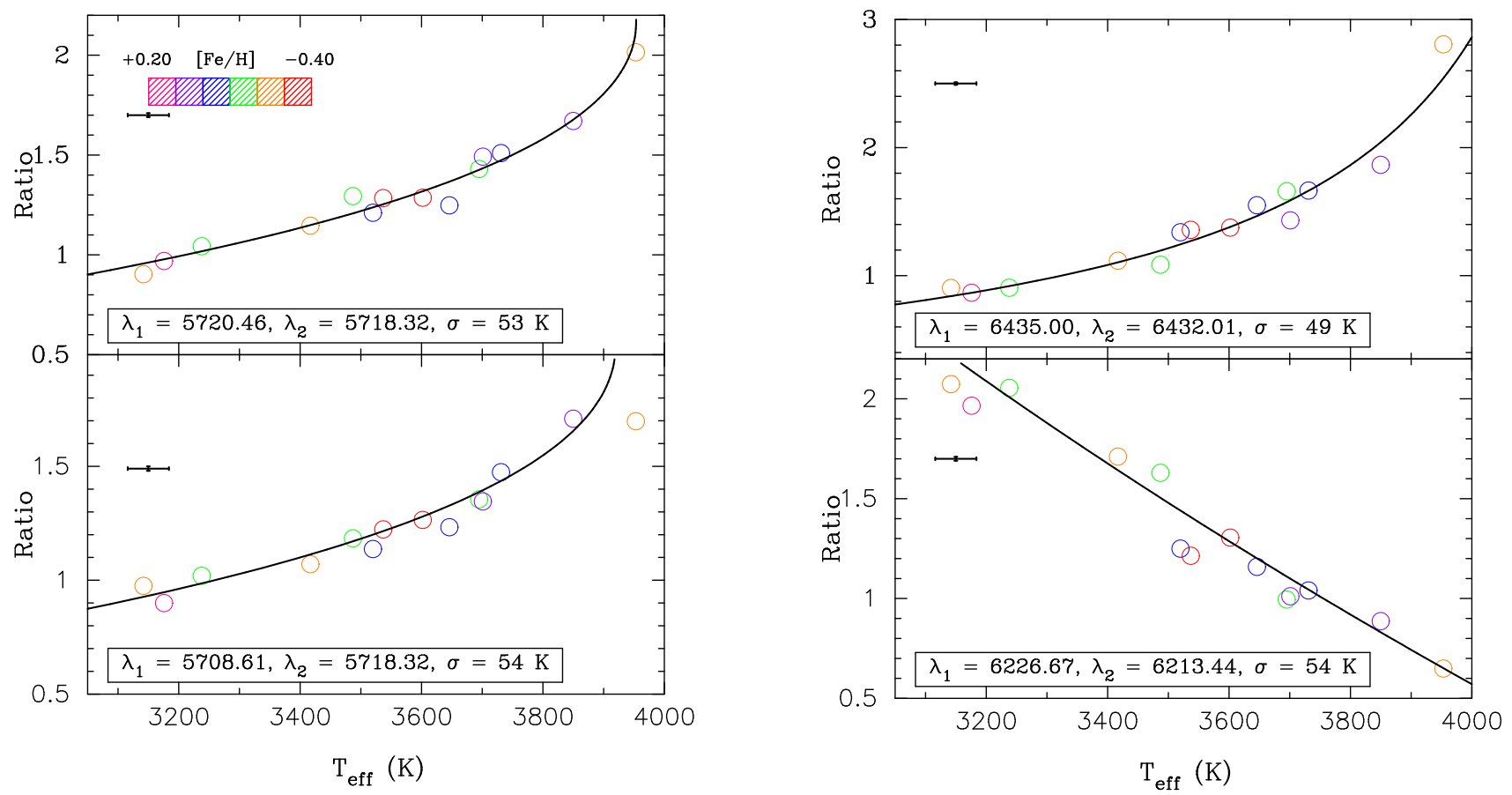

Fig. 3. Examples of ratios of some features identified to be sensitive to $T_{\text {eff }}$ in early-M dwarfs. Stars are plotted using different colours according to their metallicity (using bins of 0.10 dex length). Median uncertainties are shown in the left upper corner of each plot. The corresponding fits are also plotted. The features' central wavelengths as well as the rms standard deviation of the residuals are given in each plot.

Table 3. Spectral type calibration sample.

\begin{tabular}{|c|c|c|c|c|c|}
\hline GJ & SpT & $\operatorname{Ref}^{\dagger}$ & GJ & SpT & $\operatorname{Ref}^{\dagger}$ \\
\hline 185 & K7V & $\mathrm{h}$ & 408 & M2V & $\mathrm{h}$ \\
\hline 686 & M0V & $\mathrm{h}$ & $250 \mathrm{~B}$ & $\mathrm{M} 2.5 \mathrm{~V}$ & $\mathrm{k}$ \\
\hline 701 & M0V & $\mathrm{h}$ & 581 & $\mathrm{M} 2.5 \mathrm{~V}$ & $\mathrm{~h}$ \\
\hline 846 & $\mathrm{M} 0.5 \mathrm{~V}$ & $\mathrm{k}$ & $352 \mathrm{~A}$ & M3V & $\mathrm{k}$ \\
\hline $720 \mathrm{~A}$ & $\mathrm{M} 0.5 \mathrm{~V}$ & $\mathrm{k}$ & 436 & M3V & $\mathrm{k}$ \\
\hline 229 & M1V & $\mathrm{k}$ & $752 \mathrm{~A}$ & M3V & $\mathrm{k}$ \\
\hline $412 \mathrm{~A}$ & M1V & $\mathrm{h}$ & $569 \mathrm{~A}$ & M3V & $\mathrm{k}$ \\
\hline 514 & M1V & $\mathrm{h}$ & 273 & M3.5V & $\mathrm{k}$ \\
\hline $570 B$ & M1V & $\mathrm{h}$ & 643 & M3.5V & $\mathrm{k}$ \\
\hline 908 & M1V & $\mathrm{h}$ & 734B & M3.5V & $\mathrm{k}$ \\
\hline 205 & M1.5V & $\mathrm{k}$ & 213 & M4V & $\mathrm{k}$ \\
\hline $15 \mathrm{~A}$ & M1.5V & $\mathrm{h}$ & 402 & M4V & $\mathrm{k}$ \\
\hline 526 & M1.5V & $\mathrm{h}$ & 699 & M4V & $\mathrm{k}$ \\
\hline 625 & M1.5V & $\mathrm{h}$ & 83.1 & M4.5 & $\mathrm{k}$ \\
\hline 220 & M2V & $\mathrm{k}$ & $166 \mathrm{C}$ & M4.5 & $\mathrm{k}$ \\
\hline 382 & M2V & $\mathrm{k}$ & $234 \mathrm{~A}$ & M4.5 & $\mathrm{k}$ \\
\hline 393 & M2V & $\mathrm{h}$ & & & \\
\hline
\end{tabular}

Notes. ${ }^{(\dagger)}$ k: standard from Kirkpatrick et al. (1991); h: standard from Henry et al. (1994).

\subsection{Spectral types}

A similar approach was followed to derive spectral types. HARPS or HARPS-N spectra were obtained for a sample of 33 stars with homogeneously derived spectral types in Kirkpatrick et al. (1991) and Henry et al. (1994). The sample contains stars with spectral types between K7V and M4.5V. These stars are listed in Table 3.

Spectral type-sensitive ratios were identified by means of a Spearman correlation test. For each of them, a third-order polynomial fit was performed between the numerical spectral-type index (with value 0.0 for M0; 0.5 for M0.5 and so on) and the
Table 4. Coefficients of our spectral type calibrations.

\begin{tabular}{ccccccc}
\hline \hline $\begin{array}{c}\lambda_{1} \\
(1)\end{array}$ & $\begin{array}{c}\lambda_{2} \\
(2)\end{array}$ & $\begin{array}{c}a_{0} \\
(3)\end{array}$ & $\begin{array}{c}a_{1} \\
(4)\end{array}$ & $\begin{array}{c}a_{2} \\
(5)\end{array}$ & $\begin{array}{c}a_{3} \\
(6)\end{array}$ & $\begin{array}{c}\sigma \\
(7)\end{array}$ \\
\hline 5468.06 & 5481.92 & -3.88 & 10.79 & -4.25 & 0.48 & 0.49 \\
5627.63 & 5667.53 & 5.11 & -0.75 & -2.18 & 0.50 & 0.41 \\
5467.06 & 5481.92 & -3.80 & 8.18 & -0.56 & -0.77 & 0.48 \\
5467.06 & 5512.54 & -5.09 & 10.67 & -4.13 & 0.53 & 0.45 \\
\hline
\end{tabular}

Notes. Columns (1) and (2) show the wavelength of the corresponding features; Cols. (3) to (6) the coefficients of the fit; while Col. (7) gives the standard deviation of the calibration of spectral types. The full table is available at the CDS.

ratio. A negative value implies that the star is a late- $K$ dwarf instead of an $\mathrm{M}$ star because the index value for $\mathrm{K} 7$ is equal to -1.0 (K7 is the subtype preceding M0).

The third-order polynomial fit was preferred amongst other functional fits since we found it to give the lowest rms standard deviation. Our final selection of spectral-type-sensitive ratios includes 82 ratios of features with a standard deviation lower than 0.5 spectral subtypes. The derived mean numerical spectral types are rounded to the nearest half integer. Some examples are shown in Fig. 4. Full details for some examples are given in Table 4. We performed 1000 simulations with random spectral types. The results show that we are not able to find any suitable calibration in any of the simulations when random spectral types are used.

\subsection{Metallicity}

A common approach to find metallicity calibrators for low-mass stars relies on the search of $\mathrm{M}$ dwarfs in common proper motion pairs orbiting a solar-type star with accurate spectroscopic metallicity determinations. The basic assumption is that both 

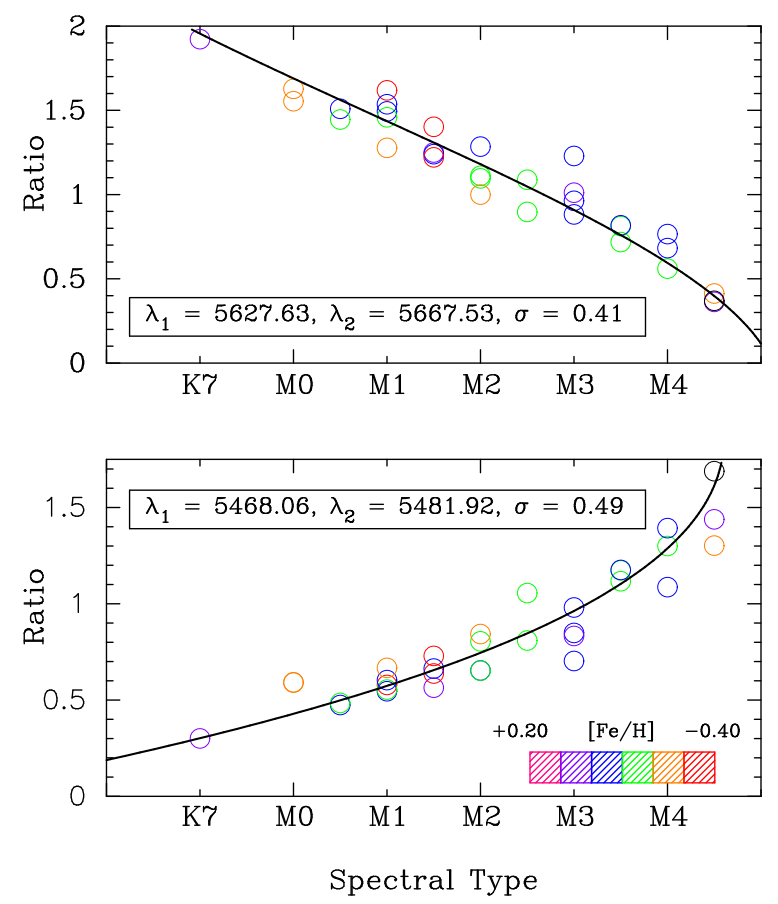

Fig. 4. Spectral type as a function of two different spectral-type sensitive ratios. Stars are plotted using different colours according to their metallicity (using 0.10 dex length bins). A third-order polynomial fit is shown. The features' central wavelengths as well as the rms standard deviation on spectral type of the residuals are given in each plot.

stars are coeval and born in the same protostellar cloud so the metallicity of the secondary $\mathrm{M}$ dwarf is the same as that of the primary star (e.g. Bonfils et al. 2005). Until very recently, only the most nearby and bright $\mathrm{M}$ stars have been searched for planets by means of the Doppler technique. As a consequence, there is a lack of HARPS and HARPS-N spectra for most of the identified $\mathrm{M}$ dwarfs in binary systems around solar-type stars. To overcome this difficulty, we assembled a list of 47 metallicity calibrators with available HARPS spectra, known parallaxes, and magnitudes by using the most recent photometric calibration provided in NE12. This calibration is in turn based on metallicity determinations from FGK primaries with an M dwarf secondary. The sample of metallicity calibrators covers a wide range in metallicity from -0.54 to +0.24 dex with typical error bars of $\lesssim 0.05$ dex. These stars are listed in Table 5 . We caution that the uncertainties reported in Table 5 do not take into account the scatter in the NE12 calibration, which is of $\sim 0.17$ dex.

The effects of metallicity on the EW of the features are entangled with the effects of $T_{\text {eff }}$, with $T_{\text {eff }}$ as the primary driver of changes in the EW. This can be easily seen in the histograms in Fig. 5. They show the distribution of the Spearman rank correlation factor of the EW with the stellar metallicity and with $T_{\text {eff }}$. The figure shows that a significant fraction of the features, $\sim 43 \%$, shows a high (Spearman correlation factor $<-0.80$ ) anticorrelation with $T_{\text {eff }}$, while only a relatively small fraction ( $\left.3 \%\right)$ shows a significant positive correlation. The correlation between stellar metallicity and EWs is generally less significant. The distribution of Spearman coefficients for metallicity shows a clear peak at +0.25 that drops almost to zero at +0.50 , while at negative values it has a smooth tail down to -0.80 . Effects of metallicity and effective temperature should therefore be considered simultaneously.

We searched for empirical relationships for metallicity as a function of features and ratios of features (i.e., an indicator of
Table 5. Metallicity calibration sample. $[\mathrm{Fe} / \mathrm{H}]$ are computed from $V$ magnitudes and parallaxes taken from the compilation of Bonfils et al. (2013) and 2MASS magnitudes (Cutri et al. 2003).

\begin{tabular}{lr|lr}
\hline \hline Star & {$[\mathrm{Fe} / \mathrm{H}]$} & Star & {$[\mathrm{Fe} / \mathrm{H}]$} \\
\hline GJ1 & $-0.37 \pm 0.04$ & GJ551 & $0.13 \pm 0.04$ \\
GJ105B & $-0.13 \pm 0.03$ & GJ555 & $0.10 \pm 0.04$ \\
GJ176 & $0.02 \pm 0.04$ & GJ569A & $0.02 \pm 0.03$ \\
GJ205 & $-0.03 \pm 0.19$ & GJ588 & $0.05 \pm 0.03$ \\
GJ2066 & $-0.10 \pm 0.03$ & GJ618A & $-0.06 \pm 0.04$ \\
GJ213 & $-0.24 \pm 0.04$ & GJ628 & $-0.05 \pm 0.03$ \\
GJ229 & $-0.02 \pm 0.17$ & GJ674 & $-0.20 \pm 0.03$ \\
GJ250B & $-0.10 \pm 0.04$ & GJ678.1A & $-0.14 \pm 0.04$ \\
GJ273 & $-0.11 \pm 0.03$ & GJ680 & $-0.05 \pm 0.04$ \\
GJ300 & $0.06 \pm 0.03$ & GJ682 & $0.10 \pm 0.03$ \\
GJ357 & $-0.32 \pm 0.03$ & GJ686 & $-0.30 \pm 0.03$ \\
GJ358 & $0.05 \pm 0.03$ & GJ693 & $-0.29 \pm 0.03$ \\
GJ367 & $-0.05 \pm 0.04$ & GJ701 & $-0.19 \pm 0.03$ \\
GJ382 & $0.05 \pm 0.03$ & GJ752A & $0.01 \pm 0.03$ \\
GJ393 & $-0.11 \pm 0.04$ & GJ832 & $-0.17 \pm 0.04$ \\
GJ413.1 & $-0.11 \pm 0.04$ & GJ846 & $-0.07 \pm 0.04$ \\
GJ438 & $-0.51 \pm 0.07$ & GJ849 & $0.24 \pm 0.04$ \\
GJ447 & $-0.26 \pm 0.03$ & GJ876 & $0.13 \pm 0.03$ \\
GJ465 & $-0.54 \pm 0.04$ & GJ877 & $-0.02 \pm 0.03$ \\
GJ479 & $0.05 \pm 0.04$ & GJ887 & $-0.35 \pm 0.14$ \\
GJ514 & $-0.11 \pm 0.04$ & GJ908 & $-0.39 \pm 0.03$ \\
GJ526 & $-0.16 \pm 0.03$ & HIP31293 & $0.03 \pm 0.04$ \\
GJ536 & $-0.15 \pm 0.04$ & LTT9759 & $0.14 \pm 0.04$ \\
GJ54.1 & $-0.47 \pm 0.05$ & & \\
\hline
\end{tabular}

Notes. The photometric calibration by NE12 is used. Errors are computed by propagating the uncertainties in the parallaxes and the photometry.

temperature) with the following analytical form:

$[\mathrm{Fe} / \mathrm{H}]=(A \times E W)+(B \times r)+C$,

where EW is the EW of a feature showing a strong metallicity correlation, $r$ is a temperature-sensitive ratio of features, and $A, B, C$ are independent coefficients. We considered every combination of features and ratios satisfying the condition that the correlation of EW with metallicity and the correlation of $r$ with $T_{\text {eff }}$ show at least a $98 \%$ of significance. Our final selection consists of 696 calibrations with standard deviation values between 0.07 and $\sim 0.10$ dex. We point out that these uncertainties should be considered as lower limits since they do not take into account possible systematic errors in the underlying NE12 calibration.

As before, we performed a series of simulations using random metallicities and errors. In $84 \%$ of the simulations we did not find any suitable metallicity calibration, although in $7.5 \%$ of the cases the simulation finds very many calibrations (more than 348). Some examples of our obtained metallicity calibrations are provided in Table 6.

\section{Comparison with other methods}

Our calibrations were applied to a sample of $53 \mathrm{M}$ dwarfs from the HARPS GTO M dwarf sample (Bonfils et al. 2013) for which HARPS data were obtained from the ESO Science Data Products Archive $^{8}$. Only spectra with a median signal-to-noise ratio of at least 25 were considered. For stars with more than one spectrum available, we took the one with the highest signal-to-noise ratio.

\footnotetext{
8 archive.eso.org/wdb/wdb/adp/phase3_spectral/form? phase3_collection=HARPS
} 
J. Maldonado et al.: Stellar parameters of early-M dwarfs from ratios of spectral features at optical wavelengths

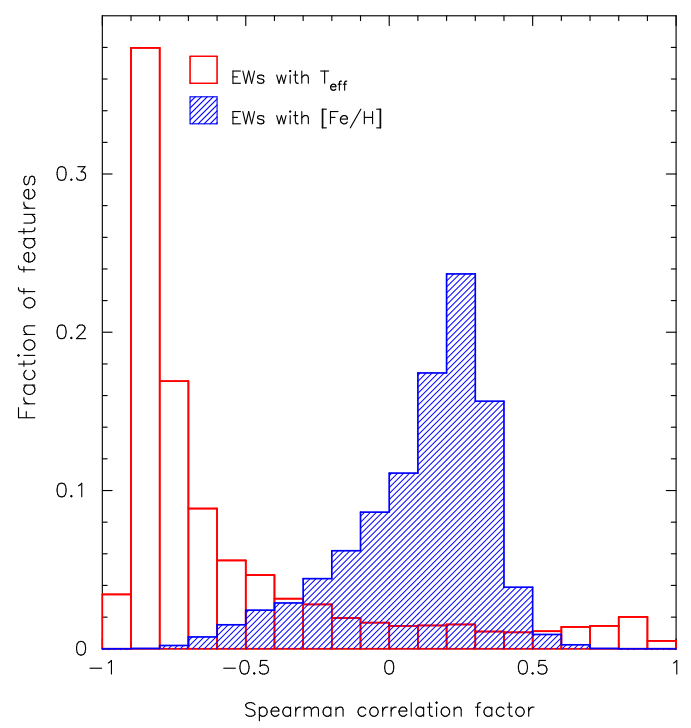

Fig. 5. Spearman correlation factor distribution of the EW with stellar metallicity (blue histogram) and with $T_{\text {eff }}$ (red histogram). The sample of metallicity calibrators and the initial list of 4224 features is considered. Metallicities are computed using NE12, whilst effective temperatures are obtained with our methodology.

Table 6. Coefficients of our metallicity calibrations.

\begin{tabular}{cccccc}
\hline \hline$\lambda_{1}$ & $\lambda_{2} / \lambda_{3}$ & $A$ & $B$ & $C$ & $\sigma(\operatorname{dex})$ \\
$(1)$ & $(2)$ & $(3)$ & $(4)$ & $(5)$ & $(6)$ \\
\hline 6785.77 & $6785.38 / 6799.25$ & -0.0354 & -1.876 & 1.753 & 0.07 \\
6785.77 & $6799.25 / 6785.38$ & -0.0349 & 0.227 & 0.416 & 0.07 \\
6785.77 & $6785.38 / 6790.93$ & -0.0359 & -0.930 & 1.633 & 0.07 \\
6785.77 & $6785.38 / 6788.76$ & -0.0375 & -1.163 & 1.581 & 0.07 \\
6785.77 & $6790.93 / 6785.38$ & -0.0356 & 0.263 & 0.613 & 0.07 \\
\hline
\end{tabular}

Notes. Column (1) shows the wavelength of the corresponding feature, Col. (2) the temperature-sensitive ratio, Cols. (3) to (5) the coefficients $A, B$, and $C$, while Col. (6) gives the standard deviation of the calibration. The full table is available at the CDS.

No further restrictions were applied. The sample is composed of nearby (distance $<11 \mathrm{pc}$ ), bright $\left(V<12, K_{\mathrm{S}}<7\right)$, early-type $\mathrm{M}$ dwarfs (spectral types M0V-M4.5V). Our methods were applied to compute effective temperatures and stellar metallicities and to derive spectral types. Final values for these parameters are the mean of the individual values from all the calibrations. All these quantities are provided in Table A.1.

Our results are compared with i) a photometric scale, namely CA08 for $T_{\text {eff }}$ and NE12 for $[\mathrm{Fe} / \mathrm{H}]$; ii) the recent work by Gaidos et al. (2014, hereafter GA14); and iii) the values obtained with the methodology developed by NE14.

\subsection{Comparison of effective temperatures}

Photometric effective temperatures were derived from $V$ magnitudes from the compilation of Bonfils et al. (2013) and 2MASS (Cutri et al. 2003) photometry using the calibration provided by CA08. Computed errors take into account the propagation of the uncertainties of the 2MASS magnitudes as well as the accuracy of the CA08 calibrations. The comparison between the photometrically derived temperatures and our spectroscopic estimates is illustrated in Fig. 6. There is a clear offset between our spectroscopic estimates and the photometric temperatures, the latter being cooler than ours (the median difference $\Delta T_{\text {eff }}=$ $T_{\mathrm{eff}}^{\mathrm{phot}}-T_{\mathrm{eff}}^{\mathrm{spec}}$ is $-198 \mathrm{~K}$ with an rms standard deviation of $176 \mathrm{~K}$ ).

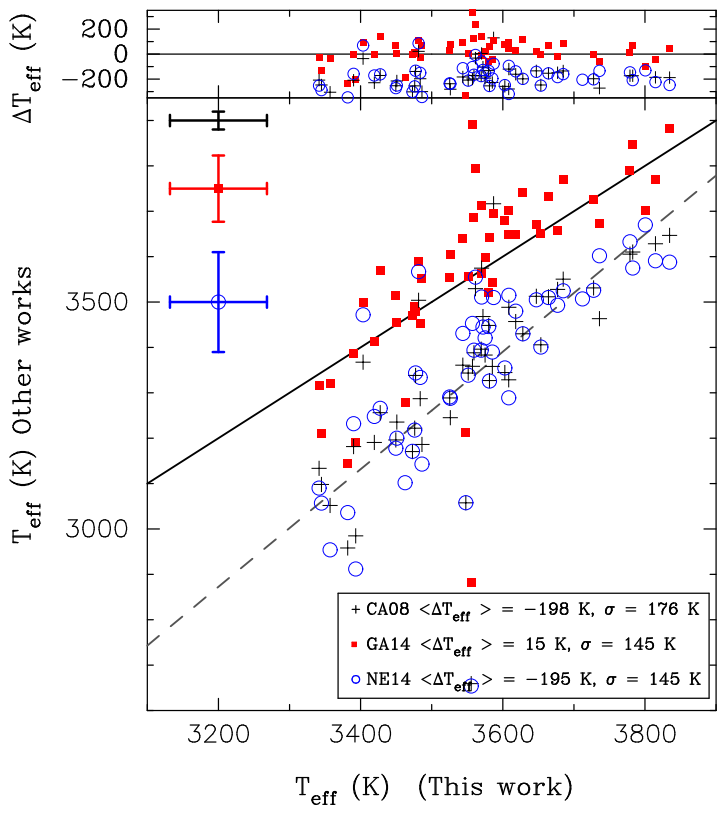

Fig. 6. $T_{\text {eff }}$ values from the literature estimates versus the values obtained in this work. Upper panel: differences between the temperatures given in the literature and the values derived in this work. Median uncertainties in the derived temperatures are also shown. The symbol \langle\rangle in the legend represents the median difference. The black continuous line represents the 1:1 relation, whilst the grey dashed line represents the best linear fit between our estimates and those obtained using the relationship of CA08 (see text in Sect. 4.1).

Our temperatures can be converted into the CA08 scale by a linear transformation: $T_{\text {eff }}[$ CA08 scale $]=(1.29 \pm 0.02) \times T_{\text {eff }}$ [this work] $+(-1271 \pm 89) \mathrm{K}$ (dashed grey line).

The reason for this discrepancy is that we chose the temperatures of Mann et al. (2013b) as our reference temperature scale. To test this, photometric $T_{\text {eff }}$ values were computed for our sample of temperature calibrators (Table 1) following CA08. The comparison between the temperatures of Mann et al. (2013b) and CA08 is shown in Fig. 7. The temperatures of CA08 tend to be systematically lower than those provided by Mann et al. (2013b).

The discrepancy between CA08 values and interferometrybased temperatures has also been noted in a recent work by Mann et al. (2015). The difference $\Delta T_{\text {eff }}$ between CA08 and interferometric-based temperatures noted by these authors is $160 \mathrm{~K}$ (the CA08 temperatures being cooler), in agreement with our results. The temperatures of CA08 were obtained by extending the IRFM for FGK dwarfs from Casagrande et al. (2006) to $\mathrm{M}$ dwarfs. One of the assumptions of the IRFM is that a star can be approximated as a blackbody for wavelengths beyond $\approx 2 \mu \mathrm{m}$. Mann et al. (2015) argued that whilst this assumption is reasonable for warmer stars, it does not suit $\mathrm{M}$ dwarfs, which have significantly more flux in the near-infrared than predicted by a blackbody. As a consequence, the temperatures of CA08 tend to be systematically lower, with increasing disparity at cooler temperatures where stars deviate more from the blackbody emission (see Figs. 6 and 7). Mann et al. (2015) also noted that the temperature scale of the old version of the PHOENIX models used in CA08 differs from interferometric-based temperatures.

We also compared our temperatures with the values given by NE14. Since the method of NE14 is calibrated using the photometric relationship of $\mathrm{CA} 08$, the comparison of our temperatures 


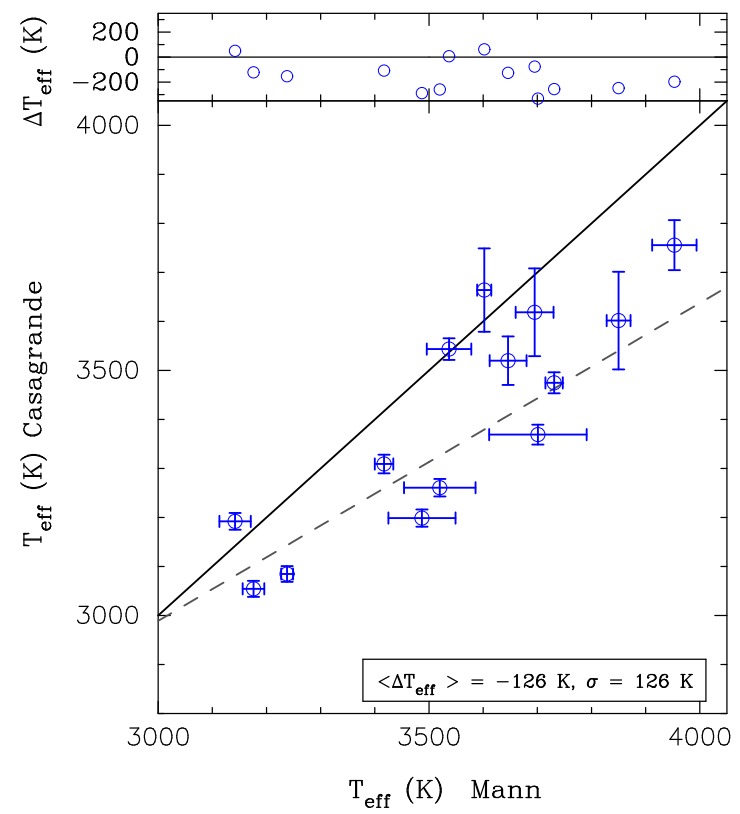

Fig. 7. $T_{\text {eff }}$ estimates based on the calibration provided by CA08 versus values from Mann et al. (2013b) and Newton et al. (2015). Upper panel: differences. The symbol \langle\rangle in the legend represents the median difference. The black continuous line represents the 1:1 relation, whilst the grey dashed line represents the best linear fit.

with NE14 shows results similar to the comparison with CA08 (Fig. 6).

Our sample contains 51 stars in common with the sample of GA14, who determined effective temperatures by comparing low-to-medium resolution spectra with the BT-SETTL version of the PHOENIX model atmospheres (Allard et al. 2012a,b). Their procedure was calibrated using the stars listed in BO12, although with the stellar bolometric fluxes computed as in Mann et al. (2013b). As can be seen in Fig. 6, the temperatures of GA14 tend to be slightly hotter than ours, especially for $T_{\text {eff }}>3400 \mathrm{~K}$. For the coolest dwarfs in this sample, temperatures of GA14 depart from ours and tend to be lower.

\subsection{Comparison of metallicities}

We also compared our metallicities with those reported previously in the literature. Values for the comparison were taken from the photometric calibration by NE12, from NE14, and from GA14, who determined metallicities following the method of Mann et al. (2013b) based on empirical calibrations between the strength of atomic and molecular spectroscopic features and stellar metallicity. The comparison is shown in Fig. 8.

The comparison reveals an overall good agreement between our metallicity estimates and those by NE12, GA14, and NE14. The median differences with these works are consistent with zero and the scatter, although somewhat large, is consistent within the (also large) error bars. A linear fit between our metallicities and those obtained using NE12 relationship provides a slope slightly steeper than one and a small difference in the zero point: $[\mathrm{Fe} / \mathrm{H}][\mathrm{NE} 12$ scale $]=(1.22 \pm 0.04) \times[\mathrm{Fe} / \mathrm{H}][$ this work $]+$ $(0.02 \pm 0.01)$ dex (dashed grey line) .

\subsection{Comparison of spectral types}

We finally compared the spectral types derived by us with those obtained by using the automatic procedure of the spectral code

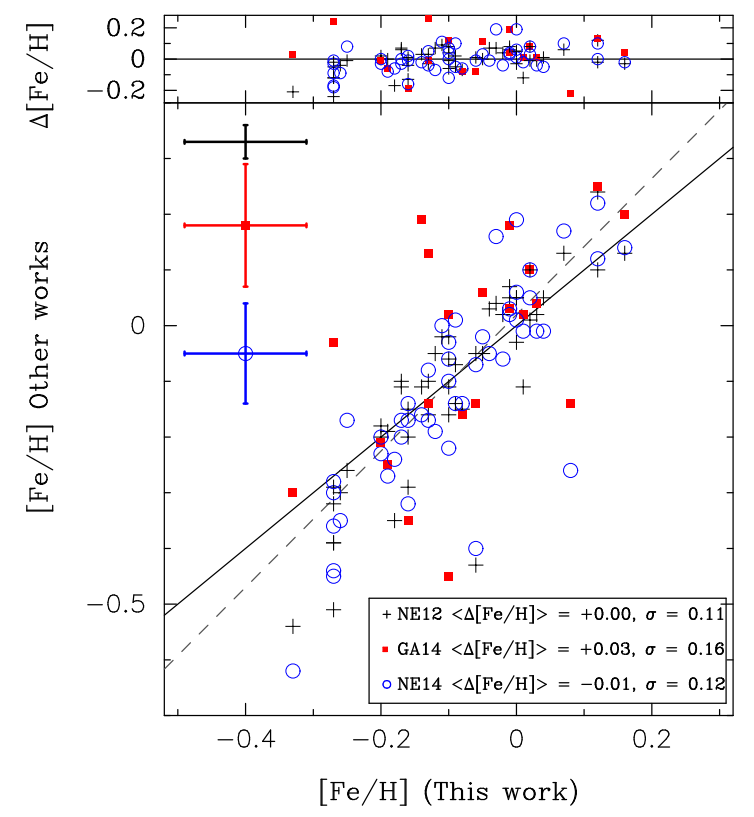

Fig. 8. $[\mathrm{Fe} / \mathrm{H}]$ values from the literature estimates versus the values obtained in this work. Upper panel: differences between the metallicities given in the literature and those derived in this work. Median uncertainties in the derived metallicities are also shown. The symbol \langle\rangle in the legend represents the median difference. The black continuous line represents the 1:1 relation, whilst the grey dashed one represents the best linear fit between our estimates and those obtained using the relationship reported by NE12 (see text in Sect. 4.2).

HAMMER (Covey et al. 2007, hereafter CO07). The code CO07 was designed to classify stars in the Sloan Digital Sky Survey spectroscopic database, before using it, our spectra were therefore degraded to a resolution $R \sim 2000$ by convolving them with a Gaussian profile. We also caution that roughly half of the spectral-type-sensitive band indices defined in $\mathrm{CO} 07$ are outside the HARPS spectral coverage. The comparison is shown in Fig. 9. There seems to be no significant difference between our estimates and those by $\mathrm{CO} 07$, with an overall good agreement within \pm 1 spectral subtype (dashed lines in Fig. 9). West et al. (2011) and Lépine et al. (2013) found the automatic spectral types given by the code HAMMER to be about one subtype earlier than the manual classification. While this effect is not evident in our comparison, it cannot be ruled out either.

Figure 10 shows our derived effective temperatures as a function of the spectral type. For comparison, data from Kenyon \& Hartmann (1995, Table A.5) are overplotted (red circles). The median $T_{\text {eff }}$-spectral type sequence from Lépine et al. (2013) is also shown (green squares). Except for some outliers, effective temperatures and spectral types are well correlated and follow the expected trend. In other words, our spectral types appear to be consistent with our temperature scale. Unlike Lépine et al. (2013), our data do not suggest a $T_{\text {eff }}$ plateau in the spectral range M1-M3, although we note that our sample is smaller than that of Lépine et al. (2013).

We conclude that our metallicities and spectral types agree reasonably well with other literature estimates. There is a clear offset between the scales of BO12 and CA08 for the effective temeperatures, as explained. In summary, our methodology can be confidently used to characterise large samples of stars in an homogeneous way. 
J. Maldonado et al.: Stellar parameters of early-M dwarfs from ratios of spectral features at optical wavelengths

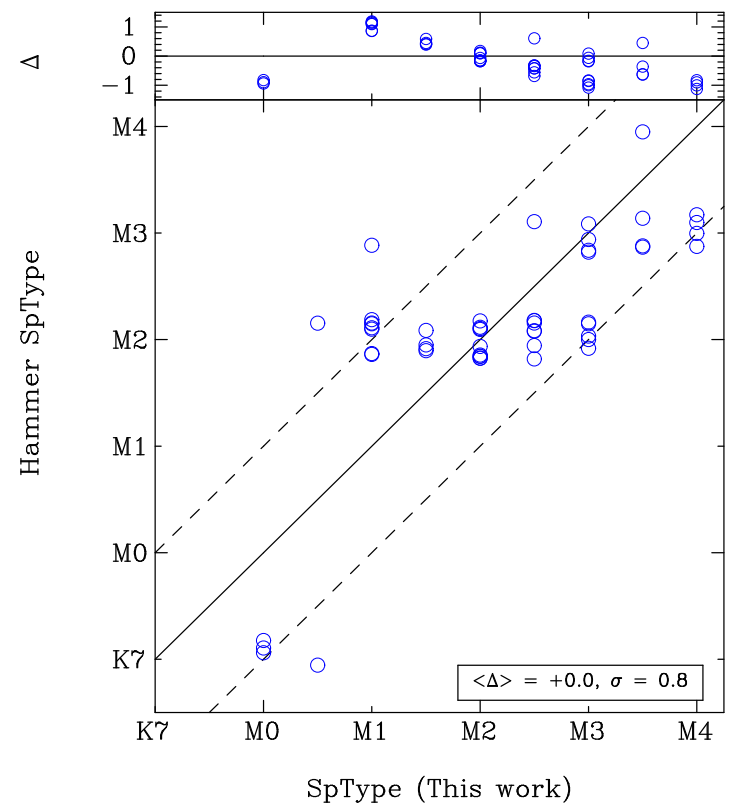

Fig. 9. M subtype values obtained by using the code HAMMER versus the values obtained in this work. Upper panel: differences with the values given in the literature. Random values between \pm 0.2 have been added to the HAMMER values to facilitate the comparison. The symbol \langle\rangle in the legend represents the median difference. The black continuous line represents the 1:1 relation, whilst the dashed lines correspond to \pm 1 spectral subtype.

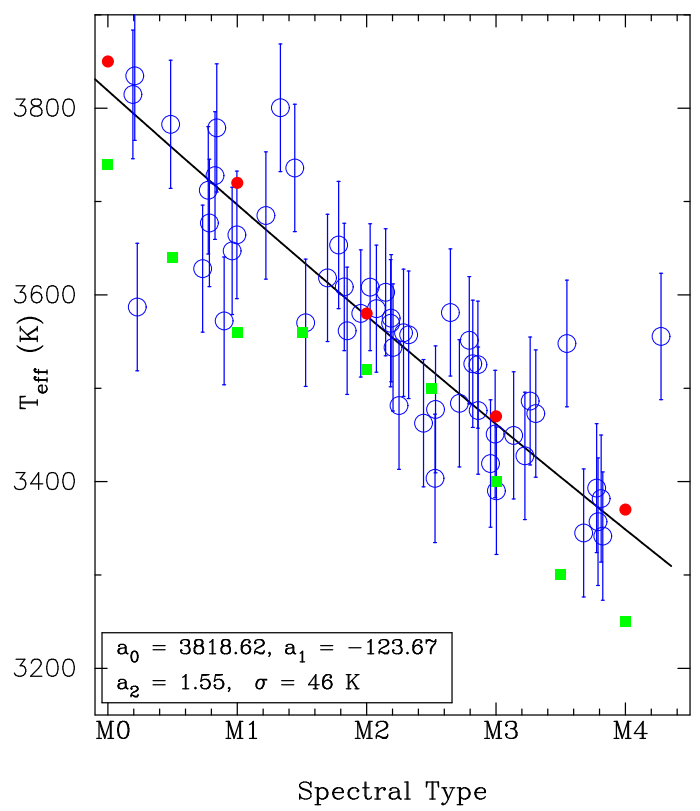

Fig. 10. Effective temperature as a function of the spectral type. For clarity the spectral types are not rounded. A second-order polynomial fit is shown. Possible outliers are removed by using a $2.5 \sigma$ clipping procedure. The coefficients of the fit as well as the rms standard deviation are given in the plot. Data from Kenyon \& Hartmann (1995, Table A.5) are overplotted using red filled circles, whilst green squares represent the median $T_{\text {eff }}$-spectral type sequence from Lépine et al. (2013).

\section{Empirical relationships for stellar mass, radius, and gravity}

We made use of the temperature and metallicity values derived with our method to search for empirical relationships with the

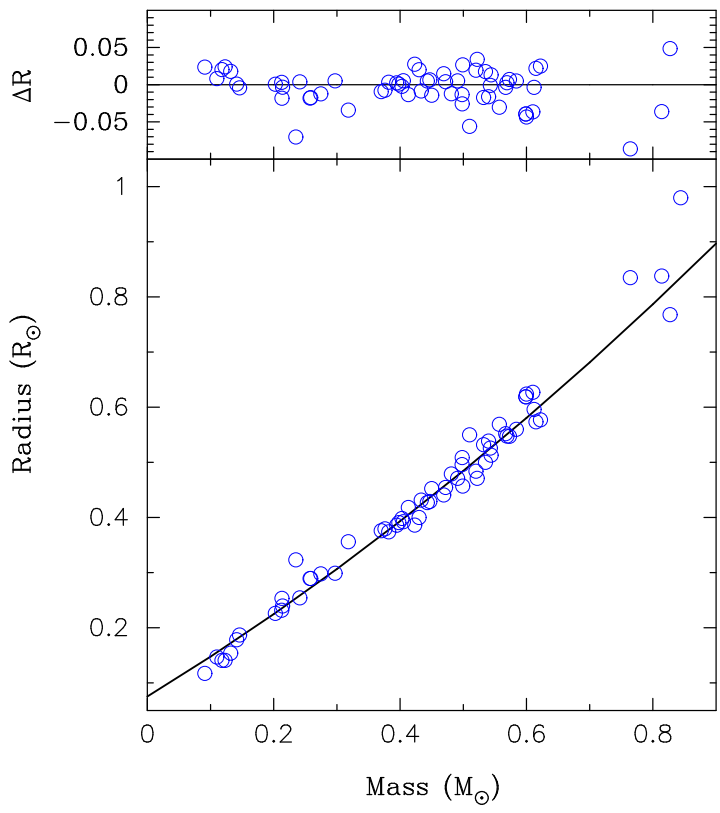

Fig. 11. Stellar radius as a function of the stellar mass. The sample includes stars with interferometric measurements of their radii from BO12 and von Braun et al. (2014) as well as low-mass eclipsing binaries from Hartman et al. (2014, Table 5). The best fit is also shown. A $3 \sigma$ clipping procedure was used to remove outliers. Upper panel: differences between the radius derived with our fit and the radius given in the literature. Median errors in radius (not shown in the plot) are of about $0.006 R_{\odot}$.

stellar evolutionary parameters namely, stellar mass, radius, and surface gravity.

We derived our own mass-radius relationship by combining the stars with known interferometric radius from BO12 and von Braun et al. (2014) with data from low-mass eclipsing binaries provided in the compilation by Hartman et al. (2014, Table 5). A $3 \sigma$ clipping procedure was used to remove potential outliers. Our derived calibration is as follows:

$R=0.0753+0.7009 \times M+0.2356 \times M^{2}$,

where radius and masses are given in solar units and the rms standard deviation of the calibration is $0.02 R_{\odot}$. The radius-mass plane is shown in Fig. 11.

Values of stellar masses were obtained for each of our target stars following the relations based on near-infrared photometry by Henry \& McCarthy (1993). We chose this calibration since it is the same as was used by BO12. These calibrations are provided in the CIT photometric system. We therefore converted the 2MASS magnitudes into CIT magnitudes before applying these calibrations, following the transformations provided by Carpenter (2001). After the stellar masses were computed, the values of the radius were derived using Eq. (4). Finally, surface gravities, $\log g$, were derived from masses and radii.

We first investigated the correlation of $M_{\star}, R_{\star}$, and $\log g$ with the effective temperature and with the stellar metallicity by using the Spearman correlation test. Results are given in Table 7. Although the main dependence of the evolutionary parameters is on the effective temperature, they also show a moderate but significant dependence on the stellar metallicity.

We also evaluated the significance of the correlations by a bootstrapp Monte Carlo (MC) test plus a Gaussian random shift of each data-point within its error bars. For each pair of variables, 10000 random datasets were created, determining the 

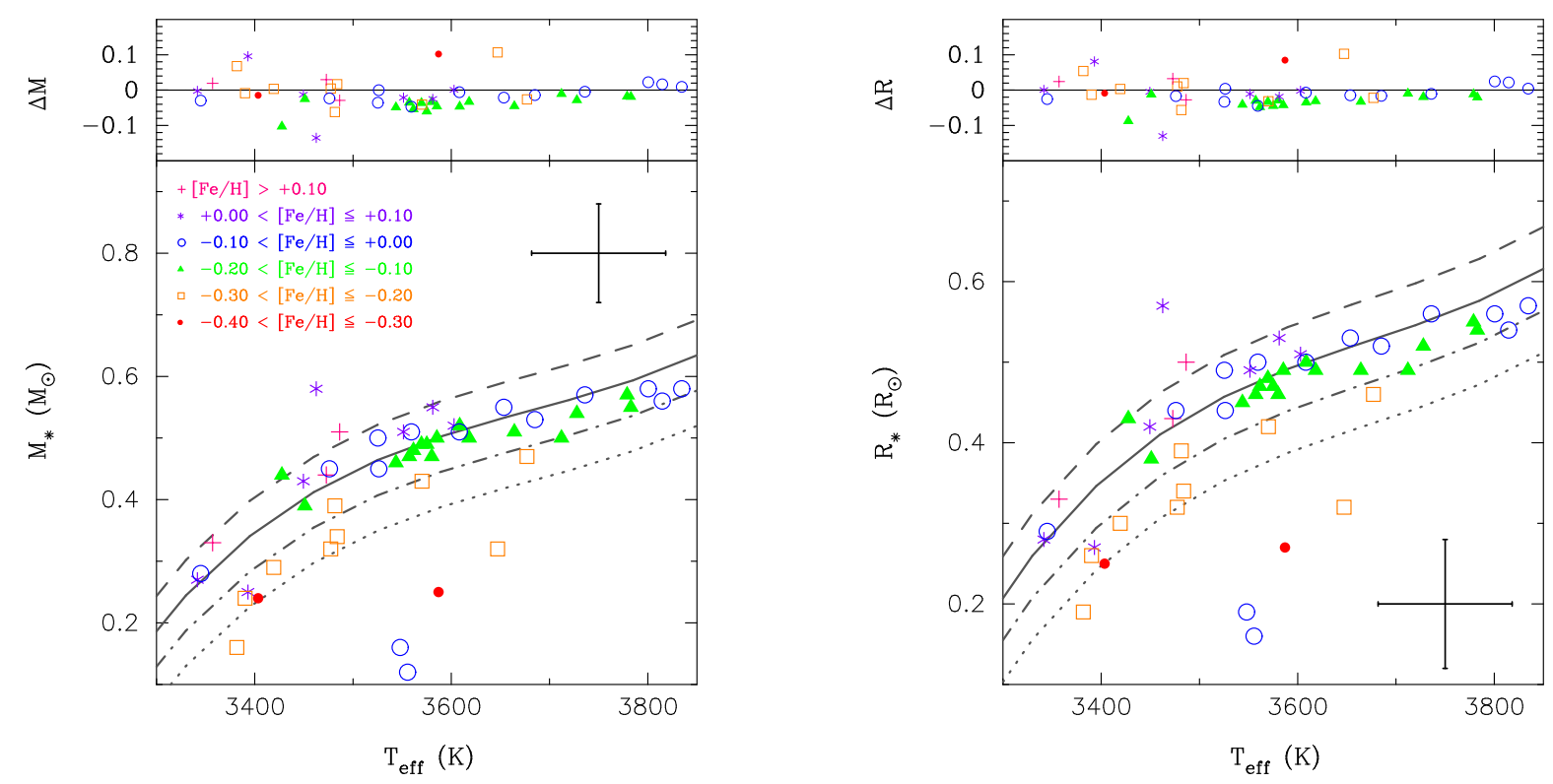

Fig. 12. Stellar mass (left panel), and radius (right panel) as a function of the effective temperature. Stars are plotted using different colours and symbols according to their metallicity. Several fits for fixed metallicity values are plotted: +0.15 (dashed line), +0.00 (solid line), -0.15 (dashed-dotted line), and -0.30 (dotted line). Upper left panel: differences between the mass obtained from Eq. (5) and those derived by using the relationship reported by Henry \& McCarthy (1993). Upper right panel: differences between the radius derived from Eq. (6) and by using Eq. (4).

Table 7. Results from the Spearman correlation test and the MC method showing the dependence of the evolutionary parameters on the effective temperature and on the stellar metallicity.

\begin{tabular}{|c|c|c|c|c|}
\hline & \multicolumn{4}{|c|}{$T_{\text {eff }}$} \\
\hline & \multicolumn{2}{|c|}{ Spearman test } & \multicolumn{2}{|c|}{ MC method } \\
\hline & $\rho$ & prob. & $\rho$ & $z$-score \\
\hline Mass & 0.72 & $2 \times 10^{-9}$ & 0.47 & 1.55 \\
\hline Radius & 0.72 & $2 \times 10^{-9}$ & 0.47 & 1.55 \\
\hline \multirow[t]{4}{*}{$\log g$} & -0.73 & $2 \times 10^{-9}$ & -0.30 & -0.93 \\
\hline & \multicolumn{4}{|c|}{$[\mathrm{Fe} / \mathrm{H}]$} \\
\hline & \multicolumn{2}{|c|}{ Spearman test } & \multicolumn{2}{|c|}{ MC method } \\
\hline & $\rho$ & prob. & $\rho$ & $z$-score \\
\hline Mass & 0.34 & 0.02 & 0.20 & 0.60 \\
\hline Radius & 0.34 & 0.02 & 0.20 & 0.60 \\
\hline $\log g$ & -0.33 & 0.02 & -0.12 & -0.40 \\
\hline
\end{tabular}

coefficient of correlation, $\rho$, and its corresponding $z$-score each time. The tests were made using the code MCSPEARman ${ }^{9}$ by Curran (2014), which might be consulted for further details on this method. Mean values of $\rho$ and $z$-score are given in Table 7. We note that the results from the MC method do not exclude a metallicity dependence, but suggest that any possible correlation is relatively weak.

A dependence of the radius on stellar metallicity is expected from model predictions (Baraffe et al. 1998; Dotter et al. 2008), but BO12 found the interferometry-based radius rather insensitive to metallicity. Furthermore, while fitting $M_{\star}, R_{\star}$, and $L_{\star}$ as a function of the effective temperature, Mann et al. (2013b) found that adding the stellar metallicity as a parameter does not improve the fits. However, in a more recent work, Mann et al. (2015) found a significant effect of the metallicity on the $T_{\text {eff }}-R_{\star}$ relation. The authors quoted small sample sizes and a sparser

\footnotetext{
9 https://github.com/PACurran/MCSpearman/
}

sampling on $[\mathrm{Fe} / \mathrm{H}]$ as the reasons why the effect of $[\mathrm{Fe} / \mathrm{H}]$ was not noticed in their previous studies. We therefore performed two fits, one using only the effective temperature and another one adding the stellar metallicity as a parameter. The extra sum-ofsquares F test (e.g. Lupton 1993) was used to test whether the addition of the metallicity to the functional form of the calibrations provides any improvement or not. The test returns a measure of the likelihood ( $p$-value) that the simpler model (the one with fewer parameters) provides a better representation than the more complicated one. The resulting values ${ }^{10}, p-M_{\star} \sim 2 \times 10^{-7}$, $p-R_{\star} \sim 7 \times 10^{-8}, p-\log g \sim 2 \times 10^{-7}$, indicate that by including the metallicity, the fits improve, in line with Mann et al. (2015). The relationships we obtain are the following:

$$
\begin{aligned}
M_{\star}\left(M_{\odot}\right)= & -171.616+0.139 \times T_{\text {eff }}-3.776 \times 10^{-5} T_{\text {eff }}^{2} \\
& +3.419 \times 10^{-9} T_{\text {eff }}^{3}+0.382 \times[\mathrm{Fe} / \mathrm{H}] \\
R_{\star}\left(R_{\odot}\right)= & -159.857+0.130 \times T_{\text {eff }}-3.534 \times 10^{-5} T_{\text {eff }}^{2} \\
& +3.208 \times 10^{-9} T_{\text {eff }}^{3}+0.347 \times[\mathrm{Fe} / \mathrm{H}] \\
\log g(\mathrm{cgs})= & 174.462-0.138 \times T_{\text {eff }}+3.728 \times 10^{-5} T_{\text {eff }}^{2} \\
& -3.376 \times 10^{-9} T_{\mathrm{eff}}^{3}-0.332 \times[\mathrm{Fe} / \mathrm{H}],
\end{aligned}
$$

where the rms standard deviations of the calibrations are $\sigma_{M \star}=$ $0.02 M_{\odot}, \sigma_{R \star}=0.02 R_{\odot}$, and $\sigma_{\log g}=0.02(\mathrm{cgs})$. The calibrations are valid for $3340 \mathrm{~K}<T_{\text {eff }}<3840 \mathrm{~K}$, and $-0.40<$ $[\mathrm{Fe} / \mathrm{H}]<+0.16$ dex. Empirical relationships for stellar luminosity are not provided since they can be easily obtained from $T_{\text {eff }}$ and $\mathrm{R}_{\star}$ by applying the Stefan-Boltzmann law. All these quantities $\left(M_{\star}, R_{\star}, \log g\right.$, and $\left.\log \left(L_{\star} / L_{\odot}\right)\right)$ for the stars analysed in this work are provided in Table A.1.

Typical uncertainties are in the order of $13.1 \%$ for the stellar mass, $11.8 \%$ for the radius, $25 \%$ for luminosities, and $0.05 \mathrm{dex}$ for $\log g$. We note that these uncertainties were computed by taking into account the $\sigma$ of the corresponding calibration and the

${ }^{10}$ The tests were performed using the MPFTEST IDL routine included in the MPFIT package (Markwardt 2009) and available at http: //cow. physics.wisc. edu/ craigm/idl/idl.html 


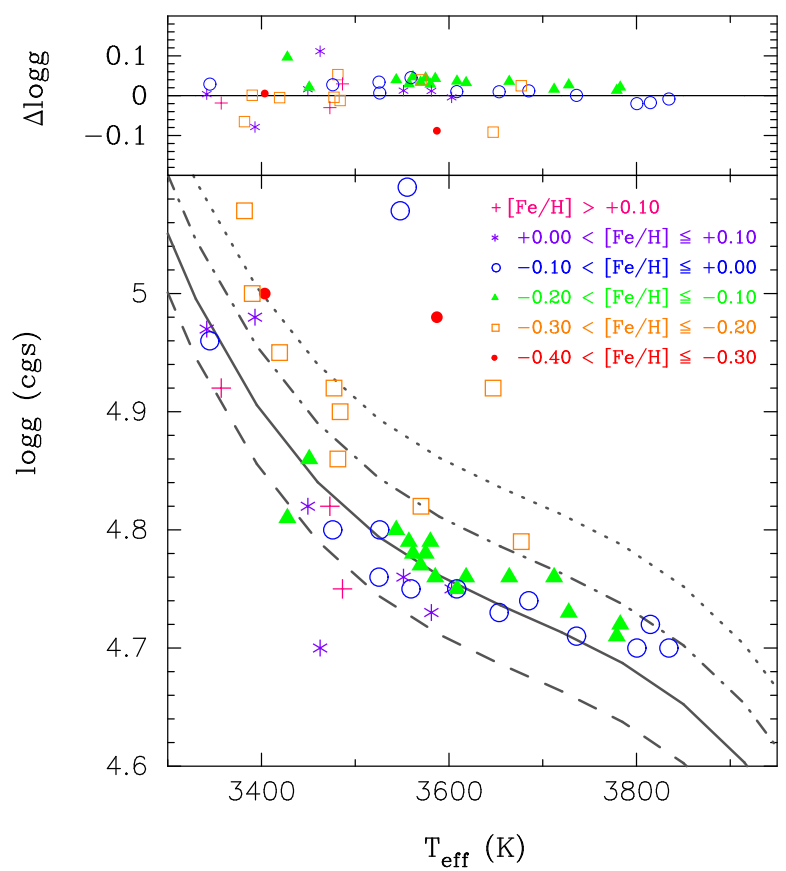

Fig. 13. Logarithmic surface gravity as a function of the effective temperature. Stars are plotted using different colours and symbols according to their metallicity. Several fits for fixed metallicity values are plotted: +0.15 (dashed line), +0.00 (solid line), -0.15 (dashed-dotted line), and -0.30 (dotted line). Upper panel: differences between the $\log g$ obtained with our calibrations (Eq. (7)) and those derived from masses and radius. Median errors in $\log g$ estimates are 0.18 dex (mass-radius derived values) and 0.05 dex (values from Eq. (7)).

propagation of the errors in $T_{\text {eff }}$ and $[\mathrm{Fe} / \mathrm{H}]$. A word of caution should be given regarding the relative errors in mass since they tend to increase towards lower masses. Relative errors in mass might be larger than $20 \%$ for stars with $M_{\star}<0.35 M_{\odot}$ and reach up to more than $40 \%$ for the few stars with $M_{\star}<0.25 M_{\odot}$. In a similar way, relative errors in radius can be larger than $20 \%$ for stars with $R_{\star}<0.35 R_{\odot}$. Relative errors in luminosities are also larger for low-luminosity stars, being significantly high (larger than $70 \%$ ) for those stars with $\log \left(L_{\star} / L_{\odot}\right)<-2$. A possible explanation may be that relative errors in masses obtained from the relationship reported in Henry \& McCarthy (1993) tend to be larger at lower masses.

The $M_{\star}$ and $R_{\star}$ versus temperature planes are shown in Fig. 12, where the stars are plotted with different colours according to their metallicities. For a given effective temperature, higher stellar metallicities predict higher masses and larger radii. Regarding surface gravity, see Fig. 13, the effect of metallicity tends to be the opposite with lower gravities in stars with higher metallicity content.

\section{Summary}

Determining accurate stellar parameters of low-mass stars is certainly a major topic in current astrophysics. This is in part because of their advantages with respect to solar-type stars in the search for small, rocky, potentially habitable planets. This fact motivated us to develop a methodology for characterising early$\mathrm{M}$ dwarfs using the high-resolution spectra that are being obtained in the current radial velocity exoplanet programmes. We made use of ratios of features as a method to determine effective temperatures and combinations of features and temperaturesensitive ratios to determine metallicities. This technique, which is frequently applied to solar-type, subgiant, and giant stars, has not been extended before to the low-mass star regime, probably because of the difficulty in identifying spectral lines in their optical spectra. We also provided empirical calibrations for masses, radii, and gravities as a function of effective temperature and metallicity. Our main results are as follows:

- The behaviour of the EW of features was studied as a function of the effective temperature and the metallicity. The results show that for a significant fraction of the features, $\sim 43 \%$, the EW shows a high anticorrelation with $T_{\text {eff }}$, whilst the correlations between EW and metallicity are in general weaker.

- Empirical calibrations for the effective temperature were obtained using stars with interferometric measurement of their radii from $\mathrm{BO} 12$ as calibrators. 112 ratios of features sensitive to the temperature were calibrated, providing effective temperatures with typical uncertainties of about $70 \mathrm{~K}$.

- In the same way, 82 ratios of features were calibrated to derive spectral types.

- Stellar metallicities were obtained from 696 combinations of EW of individual features and temperature-sensitive ratios, with estimated uncertainties in the range of 0.07-0.10 dex.

- We made use of our technique to characterise 53 early-M dwarfs that are currently being monitored in the HARPS exoplanet search programme. Photometric estimates of stellar mass, radius, and surface gravity were used to search for possible correlations of these parameters on $T_{\text {eff }}$ and $[\mathrm{Fe} / \mathrm{H}]$.

- We found stellar masses, radii, and surface gravities to have a moderate but statistically significant correlation with the stellar metallicity, in the sense that at a given effective temperature higher metallicities predict slightly higher masses and larger radii, whereas higher gravities are found in stars with lower metallicity content.

Although high-resolution HARPS and HARPS-N optical spectra were used for this work, a similar methodology can be used to derive $T_{\text {eff }}$ and $[\mathrm{Fe} / \mathrm{H}]$ for other instruments or spectral ranges.

Acknowledgements. This work was supported by the Italian Ministry of Education, University, and Research through the Premiale HARPS-N research project under grant Ricerca di pianeti intorno a stelle di piccola massa. M.P. and I.R. acknowledge financial support from the Spanish Ministry of Economy and Competitiveness (MINECO) and the Fondo Europeo de Desarrollo Regional (FEDER) through grants AYA2012-39612-C03-01 and ESP2013-48391-C4-1$\mathrm{R}$. We sincerely appreciate the careful reading of the manuscript and the constructive comments of an anonymous referee.

\section{References}

Allard, F., Homeier, D., \& Freytag, B. 2012a, Roy. Soc. London Philos. Trans. Ser. A, 370, 2765

Allard, F., Homeier, D., Freytag, B., \& Sharp, C. M. 2012b, in EAS Pub. Ser. 57, eds. C. Reylé, C. Charbonnel, \& M. Schultheis, 3

Baraffe, I., Chabrier, G., Allard, F., \& Hauschildt, P. H. 1998, A\&A, 337, 403

Bean, J. L., Benedict, G. F., \& Endl, M. 2006, ApJ, 653, L65

Biazzo, K., Frasca, A., Catalano, S., \& Marilli, E. 2007, Astron. Nachr., 328, 938

Bonfils, X., Delfosse, X., Udry, S., et al. 2005, A\&A, 442, 635

Bonfils, X., Delfosse, X., Udry, S., et al. 2013, A\&A, 549, A109

Boyajian, T. S., von Braun, K., van Belle, G., et al. 2012, ApJ, 757, 112

Caccin, B., Penza, V., \& Gomez, M. T. 2002, A\&A, 386, 286

Carpenter, J. M. 2001, AJ, 121, 2851

Casagrande, L., Portinari, L., \& Flynn, C. 2006, MNRAS, 373, 13

Casagrande, L., Flynn, C., \& Bessell, M. 2008, MNRAS, 389, 585

Cosentino, R., Lovis, C., Pepe, F., et al. 2012, in SPIE Conf. Ser., 8446, 1 
Covey, K. R., Ivezić, Ž., Schlegel, D., et al. 2007, AJ, 134, 2398 Covino, E., Esposito, M., Barbieri, M., et al. 2013, A\&A, 554, A28 Curran, P. A. 2014 [arXiv: 1411.3816]

Cutri, R. M., Skrutskie, M. F., van Dyk, S., et al. 2003, VizieR Online Data Catalog: II/246

Datson, J., Flynn, C., \& Portinari, L. 2012, MNRAS, 426, 484

Datson, J., Flynn, C., \& Portinari, L. 2014, MNRAS, 439, 1028

Datson, J., Flynn, C., \& Portinari, L. 2015, A\&A, 574, A124

Dhital, S., West, A. A., Stassun, K. G., et al. 2012, AJ, 143, 67

Dotter, A., Chaboyer, B., Jevremović, D., et al. 2008, ApJS, 178, 89

Dressing, C. D., \& Charbonneau, D. 2013, ApJ, 767, 95

Gaidos, E., Mann, A. W., Lépine, S., et al. 2014, MNRAS, 443, 2561

Gliese, W., \& Jahreiß, H. 1991, Preliminary Version of the Third Catalogue of Nearby Stars, Tech. rep.

Gray, D. F. 1989, ApJ, 347, 1021

Gray, D. F. 1994, PASP, 106, 1248

Gray, D. F., \& Johanson, H. L. 1991, PASP, 103, 439

Hartman, J. D., Bayliss, D., Brahm, R., et al. 2014, AJ, 149, 166

Henry, T. J., \& McCarthy, Jr., D. W. 1993, AJ, 106, 773

Henry, T. J., Kirkpatrick, J. D., \& Simons, D. A. 1994, AJ, 108, 1437

Johnson, J. A., \& Apps, K. 2009, ApJ, 699, 933

Kenyon, S. J., \& Hartmann, L. 1995, ApJS, 101, 117

Kirkpatrick, J. D., Henry, T. J., \& McCarthy, Jr., D. W. 1991, ApJS, 77, 417

Kovtyukh, V. V., \& Gorlova, N. I. 2000, A\&A, 358, 587

Kovtyukh, V. V., Soubiran, C., Belik, S. I., \& Gorlova, N. I. 2003, A\&A, 411, 559

Lépine, S., Rich, R. M., \& Shara, M. M. 2007, ApJ, 669, 1235

Lépine, S., Hilton, E. J., Mann, A. W., et al. 2013, AJ, 145, 102

Lupton, R. 1993, Statistics in theory and practice (Princeton University Press)
Maness, H. L., Marcy, G. W., Ford, E. B., et al. 2007, PASP, 119, 90

Mann, A. W., Brewer, J. M., Gaidos, E., Lépine, S., \& Hilton, E. J. 2013a, AJ, 145,52

Mann, A. W., Gaidos, E., \& Ansdell, M. 2013b, ApJ, 779, 188

Mann, A. W., Feiden, G. A., Gaidos, E., \& Boyajian, T. 2015, ApJ, 804, 64

Markwardt, C. B. 2009, in Astronomical Data Analysis Software and Systems XVIII, eds. D. A. Bohlender, D. Durand, \& P. Dowler, ASP Conf. Ser., 411, 251

Mayor, M., Pepe, F., Queloz, D., et al. 2003, The Messenger, 114, 20

Montes, D., Martínez-Arnáiz, R. M., Maldonado, J., et al. 2007, Highlights of Astronomy, 14, 598

Neves, V., Bonfils, X., Santos, N. C., et al. 2012, A\&A, 538, A25

Neves, V., Bonfils, X., Santos, N. C., et al. 2014, A\&A, 568, A121

Newton, E. R., Charbonneau, D., Irwin, J., et al. 2014, AJ, 147, 20

Newton, E. R., Charbonneau, D., Irwin, J., \& Mann, A. W. 2015, ApJ, 800, 85

Önehag, A., Heiter, U., Gustafsson, B., et al. 2012, A\&A, 542, A33

Rajpurohit, A. S., Reylé, C., Allard, F., et al. 2014, A\&A, 564, A90

Rojas-Ayala, B., Covey, K. R., Muirhead, P. S., \& Lloyd, J. P. 2012, ApJ, 748, 93

Schlaufman, K. C., \& Laughlin, G. 2010, A\&A, 519, A105

Ségransan, D., Kervella, P., Forveille, T., \& Queloz, D. 2003, A\&A, 397, L5

Sousa, S. G., Alapini, A., Israelian, G., \& Santos, N. C. 2010, A\&A, 512, A13

Sozzetti, A., Bernagozzi, A., Bertolini, E., et al. 2013, Eur. Phys. J. Web Conf., 47,3006

Strassmeier, K. G., \& Schordan, P. 2000, Astron. Nachr., 321, 277

Terrien, R. C., Mahadevan, S., Bender, C. F., et al. 2012, ApJ, 747, L38

von Braun, K., Boyajian, T. S., van Belle, G. T., et al. 2014, MNRAS, 438, 2413

West, A. A., Morgan, D. P., Bochanski, J. J., et al. 2011, AJ, 141, 97

Woolf, V. M., \& Wallerstein, G. 2005, MNRAS, 356, 963 
J. Maldonado et al.: Stellar parameters of early-M dwarfs from ratios of spectral features at optical wavelengths

\section{Appendix A: Additional Table}

Table A.1 lists all the stars analysed in this work. The table provides star identifier (Col. 1), effective temperature in kelvin (Col. 2), spectral type (Col. 3), stellar metallicity in dex (Col. 4), logarithm of the surface gravity, $\log g$, in $\mathrm{cm} \mathrm{s}^{-2}$ (Col. 5), stellar mass in solar units (Col. 6), stellar radius in solar units (Col. 7), and stellar luminosity, $\log \left(L_{\star} / L_{\odot}\right)(\mathrm{Col} .8)$. Each measured quantity is accompanied by its corresponding uncertainty.

Table A.1. Stellar parameters.

\begin{tabular}{|c|c|c|c|c|c|c|c|}
\hline Star & $\begin{array}{l}T_{\text {eff }} \\
(\mathrm{K}) \\
(2)\end{array}$ & $\begin{array}{c}\text { Sp-Type } \\
\text { (3) }\end{array}$ & $\begin{array}{c}{[\mathrm{Fe} / \mathrm{H}]} \\
(\mathrm{dex}) \\
(4)\end{array}$ & $\begin{array}{c}\log g \\
\text { (cgs) } \\
(5) \\
\end{array}$ & $\begin{array}{c}M_{\star} \\
\left(M_{\odot}\right) \\
(6) \\
\end{array}$ & $\begin{array}{c}R_{\star} \\
\left(R_{\odot}\right) \\
(7) \\
\end{array}$ & $\begin{array}{c}\log \left(L_{\star} / L_{\odot}\right) \\
(8)\end{array}$ \\
\hline Gl1 & $3482 \pm 68$ & M2.5 & $-0.27 \pm 0.09$ & $4.91 \pm 0.06$ & $0.33 \pm 21.39 \%$ & $0.33 \pm 18.84 \%$ & $-1.834 \pm 38.49 \%$ \\
\hline G187 & $3562 \pm 68$ & M2 & $-0.16 \pm 0.09$ & $4.83 \pm 0.05$ & $0.43 \pm 13.22 \%$ & $0.42 \pm 11.99 \%$ & $-1.590 \pm 25.18 \%$ \\
\hline G1176 & $3603 \pm 68$ & M2 & $0.03 \pm 0.09$ & $4.75 \pm 0.04$ & $0.52 \pm 10.07 \%$ & $0.51 \pm 9.31 \%$ & $-1.409 \pm 20.08 \%$ \\
\hline Gl191 & $3587 \pm 68$ & M0 & $-0.39 \pm 0.09$ & $4.89 \pm 0.05$ & $0.35 \pm 15.26 \%$ & $0.35 \pm 13.64 \%$ & $-1.728 \pm 28.31 \%$ \\
\hline Gl205 & $3800 \pm 68$ & M1.5 & $0.00 \pm 0.09$ & $4.68 \pm 0.05$ & $0.60 \pm 9.35 \%$ & $0.58 \pm 8.99 \%$ & $-1.194 \pm 19.36 \%$ \\
\hline G1229 & $3779 \pm 69$ & M1 & $-0.10 \pm 0.09$ & $4.72 \pm 0.05$ & $0.55 \pm 9.81 \%$ & $0.54 \pm 9.34 \%$ & $-1.276 \pm 20.04 \%$ \\
\hline HIP31293 & $3526 \pm 68$ & M3 & $-0.04 \pm 0.09$ & $4.81 \pm 0.05$ & $0.45 \pm 13.63 \%$ & $0.44 \pm 12.39 \%$ & $-1.564 \pm 25.95 \%$ \\
\hline Gl250B & $3557 \pm 68$ & M2.5 & $-0.13 \pm 0.09$ & $4.82 \pm 0.05$ & $0.44 \pm 13.08 \%$ & $0.43 \pm 11.88 \%$ & $-1.576 \pm 24.97 \%$ \\
\hline Gl273 & $3342 \pm 69$ & M4 & $0.01 \pm 0.09$ & $4.97 \pm 0.11$ & $0.27 \pm 44.09 \%$ & $0.28 \pm 37.91 \%$ & $-2.055 \pm 76.27 \%$ \\
\hline GJ2066 & $3575 \pm 68$ & M2 & $-0.17 \pm 0.09$ & $4.82 \pm 0.05$ & $0.43 \pm 12.75 \%$ & $0.43 \pm 11.59 \%$ & $-1.577 \pm 24.40 \%$ \\
\hline Gl341 & $3783 \pm 69$ & M0.5 & $-0.16 \pm 0.09$ & $4.74 \pm 0.05$ & $0.53 \pm 10.27 \%$ & $0.52 \pm 9.75 \%$ & $-1.305 \pm 20.81 \%$ \\
\hline Gl357 & $3477 \pm 68$ & M2.5 & $-0.27 \pm 0.09$ & $4.92 \pm 0.06$ & $0.32 \pm 21.83 \%$ & $0.33 \pm 19.21 \%$ & $-1.844 \pm 39.21 \%$ \\
\hline Gl358 & $3450 \pm 68$ & M3 & $0.04 \pm 0.09$ & $4.84 \pm 0.07$ & $0.42 \pm 18.71 \%$ & $0.42 \pm 16.86 \%$ & $-1.660 \pm 34.63 \%$ \\
\hline G1367 & $3559 \pm 68$ & M2.5 & $-0.06 \pm 0.09$ & $4.80 \pm 0.05$ & $0.46 \pm 12.22 \%$ & $0.46 \pm 11.15 \%$ & $-1.525 \pm 23.59 \%$ \\
\hline Gl382 & $3653 \pm 68$ & M2 & $-0.01 \pm 0.09$ & $4.74 \pm 0.04$ & $0.53 \pm 9.48 \%$ & $0.52 \pm 8.82 \%$ & $-1.372 \pm 19.15 \%$ \\
\hline Gl388 & $3473 \pm 68$ & M3.5 & $0.12 \pm 0.10$ & $4.79 \pm 0.07$ & $0.47 \pm 15.76 \%$ & $0.46 \pm 14.36 \%$ & $-1.555 \pm 29.77 \%$ \\
\hline G1393 & $3544 \pm 68$ & M2 & $-0.17 \pm 0.09$ & $4.84 \pm 0.05$ & $0.41 \pm 14.23 \%$ & $0.41 \pm 12.86 \%$ & $-1.626 \pm 26.84 \%$ \\
\hline Gl413.1 & $3570 \pm 68$ & M2 & $-0.10 \pm 0.09$ & $4.80 \pm 0.05$ & $0.45 \pm 12.21 \%$ & $0.45 \pm 11.14 \%$ & $-1.536 \pm 23.56 \%$ \\
\hline G1433 & $3618 \pm 68$ & M1.5 & $-0.13 \pm 0.09$ & $4.79 \pm 0.04$ & $0.47 \pm 11.02 \%$ & $0.46 \pm 10.13 \%$ & $-1.490 \pm 21.61 \%$ \\
\hline Gl438 & $3647 \pm 68$ & M1 & $-0.27 \pm 0.09$ & $4.83 \pm 0.04$ & $0.43 \pm 11.78 \%$ & $0.42 \pm 10.78 \%$ & $-1.548 \pm 22.82 \%$ \\
\hline G1447 & $3382 \pm 68$ & M4 & $-0.25 \pm 0.09$ & $5.00 \pm 0.09$ & $0.23 \pm 44.05 \%$ & $0.24 \pm 36.99 \%$ & $-2.156 \pm 74.41 \%$ \\
\hline G1465 & $3403 \pm 69$ & M2.5 & $-0.33 \pm 0.09$ & $5.01 \pm 0.09$ & $0.23 \pm 41.42 \%$ & $0.24 \pm 34.72 \%$ & $-2.154 \pm 69.91 \%$ \\
\hline Gl479 & $3476 \pm 68$ & M3 & $0.00 \pm 0.09$ & $4.83 \pm 0.06$ & $0.43 \pm 16.71 \%$ & $0.42 \pm 15.10 \%$ & $-1.630 \pm 31.20 \%$ \\
\hline G1514 & $3728 \pm 68$ & M1 & $-0.14 \pm 0.09$ & $4.76 \pm 0.04$ & $0.51 \pm 9.92 \%$ & $0.50 \pm 9.30 \%$ & $-1.362 \pm 20.00 \%$ \\
\hline G1526 & $3609 \pm 68$ & M2 & $-0.10 \pm 0.09$ & $4.79 \pm 0.04$ & $0.47 \pm 10.99 \%$ & $0.47 \pm 10.10 \%$ & $-1.483 \pm 21.57 \%$ \\
\hline G1536 & $3685 \pm 68$ & M1 & $-0.08 \pm 0.09$ & $4.75 \pm 0.04$ & $0.52 \pm 9.66 \%$ & $0.50 \pm 9.00 \%$ & $-1.377 \pm 19.47 \%$ \\
\hline G1551 & $3555 \pm 68$ & M4.5 & $-0.03 \pm 0.09$ & $4.79 \pm 0.05$ & $0.47 \pm 12.04 \%$ & $0.46 \pm 11.01 \%$ & $-1.511 \pm 23.29 \%$ \\
\hline Gl569A & $3608 \pm 68$ & M2 & $-0.02 \pm 0.09$ & $4.76 \pm 0.04$ & $0.50 \pm 10.32 \%$ & $0.49 \pm 9.52 \%$ & $-1.433 \pm 20.48 \%$ \\
\hline Gl581 & $3419 \pm 68$ & M3 & $-0.20 \pm 0.09$ & $4.95 \pm 0.08$ & $0.29 \pm 29.73 \%$ & $0.30 \pm 25.84 \%$ & $-1.948 \pm 52.29 \%$ \\
\hline G1588 & $3525 \pm 68$ & M3 & $0.00 \pm 0.09$ & $4.79 \pm 0.05$ & $0.46 \pm 13.21 \%$ & $0.46 \pm 12.04 \%$ & $-1.539 \pm 25.28 \%$ \\
\hline G1618A & $3451 \pm 68$ & M3 & $-0.10 \pm 0.09$ & $4.88 \pm 0.07$ & $0.37 \pm 21.30 \%$ & $0.37 \pm 18.96 \%$ & $-1.764 \pm 38.74 \%$ \\
\hline G1628 & $3345 \pm 69$ & M3.5 & $-0.05 \pm 0.09$ & $4.99 \pm 0.11$ & $0.25 \pm 46.49 \%$ & $0.26 \pm 39.59 \%$ & $-2.104 \pm 79.61 \%$ \\
\hline GJ644A & $3463 \pm 68$ & M2.5 & $0.08 \pm 0.10$ & $4.81 \pm 0.07$ & $0.45 \pm 17.19 \%$ & $0.44 \pm 15.59 \%$ & $-1.603 \pm 32.17 \%$ \\
\hline $\mathrm{Gl}^{2} 667 \mathrm{C}^{\star}$ & $3572 \pm 68$ & M1 & & & & & \\
\hline G1674 & $3484 \pm 68$ & M2.5 & $-0.20 \pm 0.09$ & $4.89 \pm 0.06$ & $0.36 \pm 19.49 \%$ & $0.36 \pm 17.32 \%$ & $-1.767 \pm 35.52 \%$ \\
\hline Gl678.1A & $3815 \pm 69$ & M0 & $-0.09 \pm 0.09$ & $4.70 \pm 0.05$ & $0.58 \pm 1$ & $0.56 \pm$ & $-1.222 \pm 20.77 \%$ \\
\hline G1680 & $3585 \pm 68$ & M2 & $-0.12 \pm 0.09$ & $4.80 \pm 0.05$ & $0.45 \pm 11.84 \%$ & $0.45 \pm 10.82 \%$ & $-1.527 \pm 22.93 \%$ \\
\hline G1682 & $3393 \pm 69$ & M4 & $0.02 \pm 0.09$ & $4.90 \pm 0.09$ & $0.35 \pm 28.14 \%$ & $0.35 \pm 24.92 \%$ & $-1.834 \pm 50.50 \%$ \\
\hline G1686 & $3677 \pm 68$ & M1 & $-0.26 \pm 0.09$ & $4.81 \pm 0.04$ & $0.44 \pm 11.23 \%$ & $0.44 \pm 10.34 \%$ & $-1.502 \pm 21.97 \%$ \\
\hline G1693 & $3390 \pm 68$ & M3 & $-0.27 \pm 0.09$ & $5.00 \pm 0.09$ & $0.23 \pm 42.13 \%$ & $0.25 \pm 35.45 \%$ & $-2.141 \pm 71.36 \%$ \\
\hline G1701 & $3664 \pm 68$ & M1 & $-0.19 \pm 0.09$ & $4.80 \pm 0.04$ & $0.46 \pm 10.75 \%$ & $0.46 \pm 9.92 \%$ & $-1.471 \pm 21.19 \%$ \\
\hline G1729 & $3548 \pm 68$ & M3.5 & $-0.06 \pm 0.10$ & $4.80 \pm 0.05$ & $0.46 \pm 13.20 \%$ & $0.45 \pm 12.04 \%$ & $-1.542 \pm 25.27 \%$ \\
\hline G1752A & $3551 \pm 68$ & M3 & $0.02 \pm 0.09$ & $4.77 \pm 0.05$ & $0.49 \pm 11.78 \%$ & $0.48 \pm 10.79 \%$ & $-1.485 \pm 22.91 \%$ \\
\hline Gl803* & $3628 \pm 68$ & M0.5 & & & & & \\
\hline G1832 & $3580 \pm 68$ & M2 & $-0.16 \pm 0.09$ & $4.82 \pm 0.05$ & $0.44 \pm 12.45 \%$ & $0.43 \pm 11.33 \%$ & $-1.562 \pm 23.91 \%$ \\
\hline G1846 & $3835 \pm 69$ & M0 & $-0.09 \pm 0.09$ & $4.69 \pm 0.05$ & $0.59 \pm 10.43 \%$ & $0.57 \pm 10.07 \%$ & $-1.194 \pm 21.39 \%$ \\
\hline G1849 & $3486 \pm 69$ & M3.5 & $0.12 \pm 0.09$ & $4.78 \pm 0.06$ & $0.48 \pm 14.39 \%$ & $0.47 \pm 13.13 \%$ & $-1.529 \pm 27.41 \%$ \\
\hline Gl876 & $3357 \pm 68$ & M4 & $0.16 \pm 0.09$ & $4.90 \pm 0.10$ & $0.35 \pm 31.71 \%$ & $0.35 \pm 28.14 \%$ & $-1.845 \pm 56.86 \%$ \\
\hline G1877 & $3428 \pm 68$ & M3 & $-0.11 \pm 0.09$ & $4.91 \pm 0.08$ & $0.34 \pm 25.11 \%$ & $0.34 \pm 22.17 \%$ & $-1.838 \pm 45.06 \%$ \\
\hline G1880 & $3736 \pm 68$ & M1.5 & $-0.01 \pm 0.09$ & $4.71 \pm 0.04$ & $0.57 \pm 9.04 \%$ & $0.55 \pm 8.55 \%$ & $-1.278 \pm 18.59 \%$ \\
\hline G1887 & $3712 \pm 68$ & M1 & $-0.18 \pm 0.09$ & $4.78 \pm 0.04$ & $0.49 \pm 10.26 \%$ & $0.48 \pm 9.57 \%$ & $-1.406 \pm 20.50 \%$ \\
\hline G1908 & $3570 \pm 68$ & M1.5 & $-0.27 \pm 0.09$ & $4.86 \pm 0.05$ & $0.39 \pm 14.23 \%$ & $0.39 \pm 12.82 \%$ & $-1.658 \pm 26.75 \%$ \\
\hline LTT9759 & $3581 \pm 68$ & M2.5 & $0.07 \pm 0.09$ & $4.74 \pm 0.05$ & $0.52 \pm 10.33 \%$ & $0.51 \pm 9.54 \%$ & $-1.413 \pm 20.54 \%$ \\
\hline
\end{tabular}

Notes. $\left.{ }^{\star}\right)$ The star falls out of the range of applicability of our metallicity calibrations. 